\title{
Statistical estimation of quadratic Rényi entropy for a stationary $m$-dependent sequence
}

\author{
David Källberg${ }^{1}$, Nikolaj Leonenko² ${ }^{2}$ Oleg Seleznjev ${ }^{1}$ \\ ${ }^{1}$ Department of Mathematics and Mathematical Statistics \\ Umeå University, SE-901 87 Umeå, Sweden, \\ 2 School of Mathematics, Cardiff University, \\ Senghennydd Road, Cardiff CF24 4YH, UK
}

\begin{abstract}
The Rényi entropy is a generalization of the Shannon entropy and is widely used in mathematical statistics and applied sciences for quantifying the uncertainty in a probability distribution. We consider estimation of the quadratic Rényi entropy and related functionals for the marginal distribution of a stationary $m$-dependent sequence. The $U$-statistic estimators under study are based on the number of $\epsilon$-close vector observations in the corresponding sample. A variety of asymptotic properties for these estimators are obtained (e.g., consistency, asymptotic normality, Poisson convergence). The results can be used in diverse statistical and computer science problems whenever the conventional independence assumption is too strong (e.g., $\epsilon$-keys in time series databases, distribution identification problems for dependent samples).
\end{abstract}

AMS 2010 subject classification: 62G05, 62G20, 62M99, 94A17

Keywords: Entropy estimation, quadratic Rényi entropy, stationary $m$-dependent sequence, interpoint distances, $U$-statistics

\section{Introduction}

Entropy is applied in information theory and statistics for characterizing the diversity or uncertainty in a probability distribution. For a continuous distribution $\mathcal{P}$ with density $p(x), x \in R^{d}$, the Rényi entropy is defined (Rényi, 1970) as

$$
h_{s}(\mathcal{P}):=\frac{1}{1-s} \log \left(\int_{R^{d}} p(x)^{s} d x\right), \quad s \neq 1
$$

Henceforth we use $\log x$ to denote the natural logarithm of $x$. The Rényi entropy $h_{s}$ is a generalization of the Shannon entropy (Shannon, 1948):

$$
h_{1}(\mathcal{P})=\lim _{s \rightarrow 1} h_{s}(\mathcal{P})=-\int_{R^{d}} p(x) \log p(x) d x .
$$

From the statistical point of view, the quadratic Rényi entropy

$$
h_{2}=h_{2}(\mathcal{P})=-\log \left(\int_{R^{d}} p(x)^{2} d x\right)
$$


is the simplest point on the Rényi spectrum $\left\{h_{s}(\mathcal{P}), s \in \mathcal{S}\right\}$, where $\mathcal{S}$ is subset of $R=R^{1}$, such that entropies exist. Note that $h_{2}=-\log \left(q_{2}\right)$, where we assume that the quadratic functional

$$
q_{2}=q_{2}(\mathcal{P}):=\int_{R^{d}} p(x)^{2} d x
$$

is well defined, and hence the point $s=2$ belongs to the set $\mathcal{S}$. More entropy generalizations are known in information theory, e.g., the Tsallis entropy (Tsallis, 1988):

$$
T_{s}(\mathcal{P}):=\frac{1}{1-s}\left(1-\int_{R^{d}} p(x)^{s} d x\right), \quad s \neq 1 .
$$

The Rényi entropy (or information) for stationary processes can be understood as that of the corresponding ergodic or marginal distributions, see, e.g., Gregorio and Iacus (2003), where the Rényi entropy is computed for a large class of ergodic diffusion processes.

Numerous applications of the Rényi entropy in information theoretic learning, statistics (e.g., classification, distribution identification problems, statistical inference), computer science (e.g., average case analysis for random databases, pattern recognition, image matching), and econometrics are discussed, e.g., in Principe (2010), Kapur (1989), Kapur and Kesavan (1992), Pardo (2006), Escolano et al. (2009), Neemuchwala et al. (2005), Ullah (1996), Baryshnikov et al. (2009), Seleznjev and Thalheim (2003, 2010), Thalheim (2000), Leonenko et al. (2008), and Leonenko and Seleznjev (2010).

Various estimators for the quadratic functional $q_{2}$ and the entropy $h_{2}$ for independent samples have been studied. Leonenko et al. (2008) obtain consistency of nearest-neighbor estimators for $h_{2}$, see also Penrose and Yukich (2011) and the references therein. Bickel and Ritov (1988) and Giné and Nickl (2008) show rate optimality, efficiency, and asymptotic normality of kernel-based estimators for $q_{2}$ in the one-dimensional case. Laurent (1996) builds an efficient and asymptotically normal estimator of $q_{2}$ (and more general functionals) for multidimensional distributions using orthogonal projection. See also references in these papers for more studies under the independence assumption.

In our paper, we study $U$-statistic estimators for $q_{2}$ and $h_{2}$ based on the number of $\epsilon$-close vector observations (or the number of small inter-point distances) in a sample from a stationary $m$ dependent sequence with marginal distribution $\mathcal{P}$. This extends further the results and approach in Leonenko and Seleznjev (2010) (see also Källberg and Seleznjev, 2012), where the same estimators are studied under independence. The number of small inter-point distances in an independent sample exhibits rich asymptotic behaviors, including, e.g., Poisson limits and asymptotic normality (see Jammalammadaka and Janson, 1986, and references therein). We show that some of the established limit results for this statistic are still valid when the sample is from a stationary $m$ dependent sequence. It should be noted that our normal limit theorems do not follow from the general theory developed for degenerate variable $U$-statistics under dependence, see, e.g., Kim et al., 2011, and references therein.

Note that the class of stationary $m$-dependent processes is quite large, see, e.g., the book of Joe (1997), where there are numerous copula constructions for $m$-dependent sequences with given marginal distribution, or Harrelson and Houdre (2003), where the class of stationary $m$-dependent infinitely divisible sequences is studied. 
First we introduce some notation. Throughout this paper, it is assumed that the sequence $\left\{X_{i}\right\}_{i=1}^{\infty}$ of random $d$-vectors is strictly stationary and $m$-dependent, i.e., $\left\{X_{b}, X_{b+1} \ldots, X_{b+s}\right\}$ and $\left\{X_{a-r}, X_{a-r+1}, \ldots, X_{a}\right\}$ are independent sets of vectors when $b-a>m$. Let $\mathcal{P}$ be the (marginal) distribution of $X_{t}$ with density $p(x), x \in R^{d}, p(x) \in L_{2}\left(R^{d}\right)$, and entropy $h_{2}(\mathcal{P})$. We write $d(x, y):=$ $\|x-y\|$ for the Euclidean distance in $R^{d}$ and define $B_{\epsilon}(x):=\{y: d(x, y) \leq \epsilon\}$ to be an $\epsilon$-ball in $R^{d}$ with center at $x$ and radius $\epsilon$. Denote by $b_{\epsilon}(d):=\epsilon^{d} b_{1}(d), b_{1}(d)=2 \pi^{d / 2} /(d \Gamma(d / 2))$, the volume of the $\epsilon$-ball. Let $X$ and $Y$ be independent and with distribution $\mathcal{P}$ and introduce the $\epsilon$-ball probability as

$$
p_{X, \epsilon}(x):=P\left\{X \in B_{\epsilon}(x)\right\} .
$$

Two vectors $x$ and $y$ are said to be $\epsilon$-close if $d(x, y) \leq \epsilon$, for some $\epsilon>0$. The $\epsilon$-coincidence probability for independent vectors is written $q_{2, \epsilon}:=P(d(X, Y) \leq \epsilon)=\mathrm{E} p_{X, \epsilon}(Y)$. Then the Rényi $\epsilon$-entropy $h_{2, \epsilon}(\mathcal{P}):=-\log q_{2, \epsilon}(\mathcal{P})$ can be used as a measure of uncertainty in $\mathcal{P}$ (see Seleznjev and Thalheim, 2008, Leonenko and Seleznjev, 2010). In what follows, let $\epsilon=\epsilon(n) \rightarrow 0$ as $n \rightarrow \infty$. Denote by $|C|$ the cardinality of the finite set $C$ and let $N_{n}$ be the random number of $\epsilon$-close observations in the sample $X_{1}, \ldots, X_{n}$,

$$
\left.N_{n}=N_{n, \epsilon}:=\mid\left\{d\left(X_{i}, X_{j}\right) \leq \epsilon, i, j=1, \ldots, n, i<j\right)\right\} \mid=\sum_{i<j} I\left(d\left(X_{i}, X_{j}\right) \leq \epsilon\right)=:\left(\begin{array}{l}
n \\
2
\end{array}\right) Q_{n},
$$

where $I(D)$ is the indicator of an event $D$. Then $Q_{n}$ is a $U$-statistic of Hoeffding with varying kernel. For a short introduction to $U$-statistics techniques, see, e.g., Serfling (2002), Koroljuk and Borovskich (1994), Lee (1990). Denote by $\stackrel{\mathrm{D}}{\rightarrow}$ and $\stackrel{\mathrm{P}}{\rightarrow}$ convergence in distribution and in probability, respectively. For a sequence of random variables $U_{n}, n \geq 1$, we write $U_{n}=\mathrm{O}_{\mathrm{P}}(1)$ as $n \rightarrow \infty$ if for any $\delta>0$ and large enough $n \geq 1$, there exists $C>0$ such that $P\left(\left|U_{n}\right|>C\right) \leq \delta$. Moreover, for a numerical sequence $v_{n}, n \geq 1$, let $U_{n}=\mathrm{O}_{\mathrm{P}}\left(v_{n}\right)$ as $n \rightarrow \infty$ if $U_{n} / v_{n}=\mathrm{O}_{\mathrm{P}}(1)$ as $n \rightarrow \infty$.

The developed technique can also be used for estimation of the corresponding entropy-type characteristics for discrete distributions (see, e.g., Leonenko and Seleznjev, 2010) and stationary $m$-dependent sequences. In this case, the applied estimator is a $U$-statistic with fixed kernel and so the problem is simplified in the way that some already established general results yield the limit properties, including consistency and asymptotic normality (see Appendix). An approach to statistical estimation of the Shannon entropy for discrete stationary $m$-dependent sequences can be found in Vatutin and Mikhailov (1995).

The remaining part of the paper is organized as follows. In Section 2 , the main results for the number of small inter-point distances $N_{n}$ and the estimators of $q_{2}$ and $h_{2}$ are presented. Numerical experiments illustrate the rate of convergence in the obtained asymptotic results. In Section 3 , we discuss applications of these results to $\epsilon$-keys in time series databases and distribution identification problems for dependent samples. Section 4 contains the proofs of the statements in Section 2. Some asymptotic properties of entropy estimation for the discrete case are given in Appendix.

\section{Main results}

We formulate the following assumption about finite dimensional distributions of the stationary sequence $\left\{X_{i}\right\}$. 
$\mathcal{A}$. The marginal density fulfills $p(x) \in L_{3}\left(R^{d}\right)$. Moreover, for each 4-tuple of distinct positive integers $\mathbf{t}=\left(t_{1}, t_{2}, t_{3}, t_{4}\right)$, the distribution of the random vector $\left(X_{t_{1}}, X_{t_{2}}, X_{t_{3}}, X_{t_{4}}\right)$ has a density $p_{\mathbf{t}}\left(x_{1}, x_{2}, x_{3}, x_{4}\right)$ in $R^{4 d}$ that satisfies

$$
g_{\mathbf{t}}\left(x_{1}, x_{2}\right):=\left(\int_{R^{2 d}} p_{\mathbf{t}}\left(x_{1}, x_{2}, x_{3}, x_{4}\right)^{2} d x_{3} d x_{4}\right)^{1 / 2} \in L_{1}\left(R^{2 d}\right) .
$$

Remark 1. (i) The integrability (1) ensures that the dependence among $\left\{X_{i}\right\}$ is weak enough. In fact, provided $p(x) \in L_{2}\left(R^{d}\right)$, it holds for an independent sequence, so assumption $\mathcal{A}$ is a generalization of the condition $p(x) \in L_{3}\left(R^{d}\right)$ used for studying the same estimators under independence (Källberg and Seleznjev, 2012).

(ii) If the density $p_{\mathbf{t}}\left(x_{1}, x_{2}, x_{3}, x_{4}\right)$ is bounded for each distinct $\mathbf{t}=\left(t_{1}, t_{2}, t_{3}, t_{4}\right)$, the following condition is sufficient for $\mathcal{A}$ : for each distinct pair $\left(t_{1}, t_{2}\right)$, let the density $p_{t_{1}, t_{2}}(x, y)$ of $\left(X_{t_{1}}, X_{t_{2}}\right)$ satisfy

$$
p_{t_{1}, t_{2}}(x, y) \in L_{1 / 2}\left(R^{2 d}\right) \text {. }
$$

Let in the following examples $\left\{Z_{i}\right\}_{i=-\infty}^{\infty}$ be a sequence of independent identically distributed (i.i.d.) normal $N(0,1)$-random variables.

Example 1. (i) Assumption $\mathcal{A}$ holds for all vector Gaussian sequences $\left\{X_{i}\right\}$. In particular, it is satisfied for the $m$-dependent moving average time series $\operatorname{MA}(m)$ generated by $\left\{Z_{i}\right\}$, i.e.,

$$
X_{t}=\theta_{0} Z_{t}+\cdots+\theta_{m} Z_{t-m}, \quad t \geq 1 .
$$

(ii) An exponential transformation of time series (2) gives a non-linear sequence

$$
X_{t}=\exp \left(\theta_{0} Z_{t}+\cdots+\theta_{m} Z_{t-m}\right), \quad t \geq 1 .
$$

The finite dimensional distributions of $\left\{X_{i}\right\}$ are the multivariate log-normal distributions (see, e.g., Kotz et al., 2000) and thus $\mathcal{A}$ is fulfilled in this case.

(iii) Let $X_{t}=Z_{t} / Z_{t+1}, t \geq 1$. Then $\left\{X_{i}\right\}$ is a stationary 1-dependent sequence, say, a Cauchy sequence. It can be shown that for $\mathbf{t}=(1,2,3,4)$,

$$
g_{\mathbf{t}}\left(x_{1}, x_{2}\right)=C \frac{\left|x_{2}\right|}{\left(1+x_{2}^{2}+x_{2}^{2} x_{1}^{2}\right)^{5 / 4}} \in L_{1}\left(R^{2}\right), \quad C>0,
$$

that is (1) is valid in this case and similarly for other t. Since also $p(x) \in L_{3}(R)$, condition $\mathcal{A}$ is satisfied.

\subsection{Asymptotic distribution of the number of small inter-point distances}

Let the expectation and variance of the number of small inter-point distances $N_{n}$ be $\mu_{n}=\mu_{n, \epsilon}:=$ $\mathrm{E} N_{n}$ and $\sigma_{n}^{2}=\sigma_{n, \epsilon}^{2}:=\operatorname{Var}\left(N_{n}\right)$, respectively. For $h=0,1, \ldots$, we introduce the characteristic $\sigma_{1, h, \epsilon}^{2}:=\operatorname{Cov}\left(p_{X, \epsilon}\left(X_{1}\right), p_{X, \epsilon}\left(X_{1+h}\right)\right)$. Let

$$
\zeta_{1, m}:=\lim _{n \rightarrow \infty} \frac{1}{n} \operatorname{Var}\left(\sum_{i=1}^{n} p\left(X_{i}\right)\right)=\operatorname{Var}\left(p\left(X_{1}\right)\right)+2 \sum_{h=1}^{m} \operatorname{Cov}\left(p\left(X_{1}\right), p\left(X_{1+h}\right)\right) .
$$


Proposition 1 Suppose that $\mathcal{A}$ holds.

(i) Then the expectation and variance of $N_{n}$ fulfill

$$
\begin{aligned}
& \mu_{n}=\left(\begin{array}{l}
n \\
2
\end{array}\right) q_{2, \epsilon}+\mathrm{o}\left(n \epsilon^{d / 2}\right), \\
& \sigma_{n}^{2}=\frac{n^{2}}{2} q_{2, \epsilon}+n^{3}\left(\sigma_{1,0, \epsilon}^{2}+2 \sum_{h=1}^{m} \sigma_{1, h, \epsilon}^{2}\right)+\mathrm{o}\left(n \epsilon^{d / 2}\right)+\mathrm{o}\left(n^{2} \epsilon^{d}\right) \text { as } n \rightarrow \infty .
\end{aligned}
$$

(ii) If $n^{2} \epsilon^{d} \rightarrow a, 0<a \leq \infty$, and $\zeta_{1, m}>0$ when $\sup _{n \geq 1}\left\{n \epsilon^{d}\right\}=\infty$, then

$$
\begin{aligned}
\mu_{n} & \sim \frac{1}{2} b_{1}(d) q_{2} n^{2} \epsilon^{d}, \\
\sigma_{n}^{2} & \sim \frac{1}{2} b_{1}(d) q_{2} n^{2} \epsilon^{d}+b_{1}(d)^{2} \zeta_{1, m} n^{3} \epsilon^{2 d} \text { as } n \rightarrow \infty .
\end{aligned}
$$

The asymptotic distribution for $N_{n}$ depends on the rate of decrease of $\epsilon(n)$. Some results for $N_{n}$ under the i.i.d. assumption (i.e., $m=0$ ) are obtained in Jammalamadaka and Janson (1986) (see also Leonenko and Seleznjev, 2010). With only additional weak conditions, we show that these results are still valid when $\left\{X_{i}\right\}$ is stationary and $m$-dependent. Let $\mu=\mu(a):=\frac{1}{2} b_{1}(d) q_{2} a$ for $a>0$.

Theorem 1 Suppose that $\mathcal{A}$ holds.

(i) If $n^{2} \epsilon^{d} \rightarrow 0$, then $N_{n} \stackrel{\mathrm{D}}{\rightarrow} 0$ as $n \rightarrow \infty$.

(ii) If $n^{2} \epsilon^{d} \rightarrow a, 0<a<\infty$, then $\mu=\lim _{n \rightarrow \infty} \mu_{n}$ and

$$
N_{n} \stackrel{\mathrm{D}}{\rightarrow} \operatorname{Po}(\mu) \text { as } n \rightarrow \infty \text {. }
$$

(iii) If $n^{2} \epsilon^{d} \rightarrow \infty$ and $n \epsilon^{d} \rightarrow a, 0 \leq a \leq \infty$, and $\zeta_{1, m}>0$ when $a=\infty$, then

$$
\left(N_{n}-\mu_{n}\right) / \sigma_{n} \stackrel{\mathrm{D}}{\rightarrow} N(0,1) \text { as } n \rightarrow \infty .
$$

Note that definition (3) implies $\zeta_{1, m} \geq 0$ with equality, e.g., if $\mathcal{P}$ is uniform.

Remark 2. The following inference procedure is discussed in Leonenko and Seleznjev (2010) for i.i.d. sequences. Let $c:=\frac{1}{2} b_{1}(d) q_{2}$. By applying Theorem1(ii) to the minimum inter-point distance $Y_{n}=\min _{1 \leq i<j \leq n}\left\|X_{i}-X_{j}\right\|$ and $\epsilon=c^{-1 / d} t^{1 / d} n^{-2 / d}$ for a fixed $t>0$, i.e., $\mu_{n} \rightarrow \mu=t$, we get

$$
P\left(c n^{2} Y_{n}^{d}>t\right)=P\left(Y_{n}>\epsilon\right)=P\left(N_{n}=0\right) \rightarrow e^{-\mu}=e^{-t} \text { as } n \rightarrow \infty .
$$

Hence $Z_{n}:=c n^{2} Y_{n}^{d}$ has asymptotically exponential distribution $\operatorname{Exp}(1)$ and an asymptotic confidence interval for the quadratic functional $q_{2}$ can be written

$$
I_{n}=\left[2 c_{1} /\left(n^{2} b_{1}(d) Y_{n}^{d}\right), 2 c_{2} /\left(n^{2} b_{1}(d) Y_{n}^{d}\right)\right]
$$

for certain positive $c_{1}, c_{2}$. 


\subsection{Estimation of the entropy-type characteristics}

We consider an estimator for the quadratic functional $q_{2}$ based on the normalized statistic $Q_{n}=$ $\left(\begin{array}{l}n \\ 2\end{array}\right)^{-1} N_{n}$, defined as

$$
\tilde{Q}_{n}=\tilde{Q}_{n, \epsilon}:=Q_{n} / b_{\epsilon}(d) .
$$

Let $\tilde{H}_{n}:=-\log \left(\max \left(\tilde{Q}_{n}, 1 / n\right)\right)$ be the corresponding estimator for the entropy $h_{2}$. The asymptotic behavior of $\tilde{Q}_{n}$ and $\tilde{H}_{n}$ depends on the rate of decreasing for $\epsilon(n)$. In the following theorem for consistency, we give two versions for different asymptotic rates of $\epsilon(n)$, with significantly weaker distribution assumptions in (ii).

\section{Theorem 2}

(i) If $\mathcal{A}$ holds and $n^{2} \epsilon^{d} \rightarrow \infty$, then

$$
\tilde{Q}_{n} \stackrel{\mathrm{P}}{\rightarrow} q_{2} \quad \text { and } \quad \tilde{H}_{n} \stackrel{\mathrm{P}}{\rightarrow} h_{2} \text { as } n \rightarrow \infty .
$$

(ii) Let $p(x) \in L_{3}\left(R^{d}\right)$ and assume that $P\left(X_{i} \neq X_{j}\right)=1$, for all $i \neq j$. If $n \epsilon^{d} \rightarrow a, 0<a \leq \infty$, then

$$
\tilde{Q}_{n} \stackrel{\mathrm{P}}{\rightarrow} q_{2} \quad \text { and } \quad \tilde{H}_{n} \stackrel{\mathrm{P}}{\rightarrow} h_{2} \text { as } n \rightarrow \infty .
$$

Let $\tilde{q}_{2, \epsilon}:=q_{2, \epsilon} / b_{\epsilon}(d)$ and $\tilde{h}_{2, \epsilon}:=-\log \left(\tilde{q}_{2, \epsilon}\right)=h_{2, \epsilon}+\log \left(b_{\epsilon}(d)\right)$. Next we show asymptotic normality properties for the estimators $\tilde{Q}_{n}$ and $\tilde{H}_{n}$ when $n$ and $\epsilon$ vary accordingly. Let $\nu:=2 q_{2} / b_{1}(d)$ and recall definition (3) of $\zeta_{1, m}$.

Theorem 3 Suppose that $\mathcal{A}$ holds and $n^{2} \epsilon^{d} \rightarrow \infty$.

(i) If $n \epsilon^{d} \rightarrow a, 0<a \leq \infty$, and $\zeta_{1, m}>0$ when $a=\infty$, then

$$
\sqrt{n}\left(\tilde{Q}_{n}-\tilde{q}_{2, \epsilon}\right) \stackrel{\mathrm{D}}{\rightarrow} N\left(0, \nu / a+4 \zeta_{1, m}\right) \quad \text { and } \quad \sqrt{n} \tilde{Q}_{n}\left(\tilde{H}_{n}-\tilde{h}_{2, \epsilon}\right) \stackrel{\mathrm{D}}{\rightarrow} N\left(0, \nu / a+4 \zeta_{1, m}\right) \text { as } n \rightarrow \infty .
$$

(ii) If $n \epsilon^{d} \rightarrow 0$, then

$$
n \epsilon^{d / 2}\left(\tilde{Q}_{n}-\tilde{q}_{2, \epsilon}\right) \stackrel{\mathrm{D}}{\rightarrow} N(0, \nu) \quad \text { and } \quad n \epsilon^{d / 2} \tilde{Q}_{n}\left(\tilde{H}_{n}-\tilde{h}_{2, \epsilon}\right) \stackrel{\mathrm{D}}{\rightarrow} N(0, \nu) \text { as } n \rightarrow \infty .
$$

To evaluate the quadratic functional $q_{2}$ and the entropy $h_{2}$, we introduce smoothness conditions for the marginal density $p(x)$. Denote by $H_{2}^{(\alpha)}(K), 0<\alpha \leq 1, K>0$, a linear space of functions in $R^{d}$ satisfying a $\alpha$-Hölder condition in $L_{2}$-norm with constant $K$, i.e., if $p(x) \in H_{2}^{(\alpha)}(K)$ and $h \in B_{1}(d)$, then

$$
\left(\int_{R^{d}}(p(x+h)-p(x))^{2} d x\right)^{1 / 2} \leq K|h|^{\alpha} .
$$

Note that (44) holds, e.g., if for some function $g(x) \in L_{2}\left(R^{d}\right)$,

$$
|p(x+h)-p(x)| \leq g(x)|h|^{\alpha} .
$$

There are different ways to define the density smoothness, e.g., by the conventional or pointwise Hölder conditions (Leonenko and Seleznjev, 2010, Källberg et al., 2012) or the Fourier characterization (Giné and Nickl, 2008).

The rate of convergence in probability can now be described in terms of the smoothness of $p(x)$. Let $L(n), n \geq 1$, be a slowly varying function as $n \rightarrow \infty$. 
Theorem 4 Let $\mathcal{A}$ hold and assume that $p(x) \in H_{2}^{(\alpha)}(K)$.

(i) Then the bias $\left|\mathrm{E} \tilde{Q}_{n}-q_{2}\right| \leq \frac{1}{2} K^{2} \epsilon^{2 \alpha}+\mathrm{o}\left(1 /\left(n \epsilon^{d / 2}\right)\right)$ as $n \rightarrow \infty$.

(ii) If $0<\alpha \leq d / 4$ and $\epsilon \sim c n^{-2 /(4 \alpha+d)}, c>0$, then

$$
\tilde{Q}_{n}-q_{2}=\mathrm{O}_{\mathrm{P}}\left(n^{-\frac{4 \alpha}{4 \alpha+d}}\right) \quad \text { and } \quad \tilde{H}_{n}-h_{2}=\mathrm{O}_{\mathrm{P}}\left(n^{-\frac{4 \alpha}{4 \alpha+d}}\right) \text { as } n \rightarrow \infty .
$$

(iii) If $\alpha>d / 4$ and $\epsilon \sim L(n) n^{-1 / d}$ and $n \epsilon^{d} \rightarrow a, 0<a \leq \infty$, then

$$
\tilde{Q}_{n}-q_{2}=\mathrm{O}_{\mathrm{P}}\left(n^{-\frac{1}{2}}\right) \quad \text { and } \quad \tilde{H}_{n}-h_{2}=\mathrm{O}_{\mathrm{P}}\left(n^{-\frac{1}{2}}\right) \text { as } n \rightarrow \infty .
$$

Remark 3. Since the aim of this paper is to provide asymptotic properties for estimation under dependence, we leave questions regarding efficiency and optimality of the obtained convergence rates for further research. Nevertheless, it could be mentioned that, in the independent one-dimensional case $(m=0, d=1)$, Bickel and Ritov (1988) show that the rates in Theorem 4 are optimal in a certain sense (see also Laurent, 1996, Giné and Nickl, 2008).

In order to make the normal limit results of Theorem 3 practical, e.g., to calculate approximate confidence intervals, the asymptotic variances have to be estimated. In particular, we need a consistent estimate of the characteristic $\zeta_{1, m}$. Assuming that $m$ is exactly known might be too strong in our non-parametric setting. However, note that $\zeta_{1, m}=\zeta_{1, r}$ for $r \geq m$, so under the less restrictive assumption that a bound $r$ for $m$ is known, we can use a consistent estimator of $\zeta_{1, r}$. To construct this estimator, for $h=0,1, \ldots$, consider the following estimator of $q_{3, h}:=\operatorname{E} p\left(X_{1}\right) p\left(X_{1+h}\right)$,

$$
U_{h, n}=U_{h, n, \epsilon_{0}}:=M_{h, n}^{-1} b_{\epsilon_{0}}(d)^{-2} \sum_{(i, j, k) \in \mathcal{E}_{h, n}} I\left(d\left(X_{i}, X_{j}\right) \leq \epsilon_{0}, d\left(X_{i+h}, X_{k}\right) \leq \epsilon_{0}\right), \quad \epsilon_{0}>0,
$$

where $\mathcal{E}_{h, n}:=\{(i, j, k): 1 \leq i \leq n-(h+1), j, k \neq i, i+h, j \neq k\}$ and the number of summands $M_{h, n}:=\left|\mathcal{E}_{h, n}\right|=(n-(h+1))(n-2)(n-3)$. Let $\epsilon_{0}=\epsilon_{0}(n) \rightarrow 0$ as $n \rightarrow \infty$.

Proposition 2 If $\mathcal{A}$ holds and $n \epsilon_{0}^{3 d} \rightarrow c, 0<c \leq \infty$, then

$$
U_{h, n} \stackrel{\mathrm{P}}{\rightarrow} q_{3, h} \text { as } n \rightarrow \infty \text {. }
$$

Remark 4. Under the conditions of Proposition 2, we have

$$
H_{3, n}:=-\frac{1}{2} \log \left(\max \left(U_{0, n}, 1 / n\right)\right) \stackrel{\mathrm{P}}{\rightarrow}-\frac{1}{2} \log \left(\int_{R^{d}} p(x)^{3} d x\right)=h_{3} \text { as } n \rightarrow \infty,
$$

and thus $H_{3, n}$ is a consistent estimator of the cubic Rényi entropy $h_{3}$.

A consistent plug-in estimator for $\zeta_{1, r}$ can be set up according to

$$
z_{1, r, n}:=U_{0, n}-\tilde{Q}_{n}^{2}+2 \sum_{h=1}^{r}\left(U_{h, n}-\tilde{Q}_{n}^{2}\right),
$$

where it is assumed that the sequence $\epsilon_{0}=\epsilon_{0}(n)$ satisfies $n \epsilon_{0}^{3 d} \rightarrow c, 0<c \leq \infty$.

Now we construct asymptotically pivotal quantities by using Theorem 3 , the smoothness of the marginal density, and variance estimators. To achieve $\sqrt{n}$-rate of convergence, an upper bound $r \geq m$ has to be available. Let $w_{r, n}^{2}:=2 \tilde{Q}_{n} /\left(n b_{\epsilon}(d)\right)+4 \max \left(z_{1, r, n}, 1 / n\right)$ be the corresponding consistent estimator of $\nu / a+4 \zeta_{1, m}$ when $n \epsilon^{d} \rightarrow a, 0<a \leq \infty$. 
Theorem 5 Let $\mathcal{A}$ hold and assume that $p(x) \in H_{2}^{(\alpha)}(K), \alpha>d / 4$, and $r \geq m$. If $\epsilon \sim L(n) n^{-1 / d}$ and $n \epsilon^{d} \rightarrow a, 0<a \leq \infty$, and $\zeta_{1, m}>0$ when $a=\infty$, then

$$
\sqrt{n}\left(\tilde{Q}_{n}-q_{2}\right) / w_{r, n} \stackrel{\mathrm{D}}{\rightarrow} N(0,1) \quad \text { and } \quad \sqrt{n} \tilde{Q}_{n}\left(\tilde{H}_{n}-h_{2}\right) / w_{r, n} \stackrel{\mathrm{D}}{\rightarrow} N(0,1) \text { as } n \rightarrow \infty .
$$

Next we apply Theorem 3(ii) to weaken the condition $\alpha>d / 4$ and therefore get asymptotic normality for less smooth cases. Additionaly, asymptotically pivotal quantities can be built even without a bound $r$ available for $m$. Note, however, that the obtained rate of convergence is slower than $\sqrt{n}$. Define a consistent estimator for $\nu$ as $u_{n}^{2}:=2 \max \left(\tilde{Q}_{n}, 1 / n\right) / b_{1}(d)$. Let $C_{\beta}:=\beta /(2-$ $\beta), 0<\beta<1$.

Theorem 6 Let $\mathcal{A}$ hold and assume that $p(x) \in H_{2}^{(\alpha)}(K)$ and $n^{2} \epsilon^{d} \rightarrow \infty$.

(i) If $\alpha>(d / 4) C_{\beta}$, for some $0<\beta<1$, and $\epsilon \sim c n^{-(2-\beta) / d}, c>0$, then

$$
n^{\beta / 2} c^{d / 2}\left(\tilde{Q}_{n}-q_{2}\right) / u_{n} \stackrel{\mathrm{D}}{\rightarrow} N(0,1) \quad \text { and } \quad n^{\beta / 2} c^{d / 2} \tilde{Q}_{n}\left(\tilde{H}_{n}-h_{2}\right) / u_{n} \stackrel{\mathrm{D}}{\rightarrow} N(0,1) \text { as } n \rightarrow \infty .
$$

(ii) If $\epsilon \sim L(n) n^{-2 / d}$, then

$$
L(n)\left(\tilde{Q}_{n}-q_{2}\right) / u_{n} \stackrel{\mathrm{D}}{\rightarrow} N(0,1) \quad \text { and } \quad L(n) \tilde{Q}_{n}\left(\tilde{H}_{n}-h_{2}\right) / u_{n} \stackrel{\mathrm{D}}{\rightarrow} N(0,1) \text { as } n \rightarrow \infty .
$$

The practical applicability of the results in this paper relies on an accurate choice of the parameter $\epsilon$. One possibility is to use the cross-validation techniques for choosing the optimal bandwidth for density estimation, see, e.g., Hart and Vieu (1990). However, the problem of finding a suitable $\epsilon$ is a topic for future research.

In the following examples, let $\left\{Z_{i}\right\}$ be a sequence of i.i.d. $N(0,1)$-variables.

Example 2. We consider estimation of the quadratic Rényi entropy $h_{2}(\mathcal{P})$ for the 2-dependent moving average $\mathrm{MA}(2)$ process $X_{t}=\theta_{0} Z_{t}+\theta_{1} Z_{t-1}+\theta_{2} Z_{t-2}$, where $\theta_{2}=\theta_{1}=\theta_{0}=1 / \sqrt{3}$. In this case $\mathcal{P}=N(0,1)$ and $h_{2}(\mathcal{P})=\log (2 \sqrt{\pi})$. We simulate $N_{\text {sim }}=500$ independent and normalized residuals $R_{n}^{(i)}:=\sqrt{n} \tilde{Q}_{n}\left(\tilde{H}_{n}-h_{2}\right) / w_{r, n}, i=1, \ldots, N_{\text {sim }}$, with $n=500, r=6$, and $\epsilon=\epsilon_{0}=1 / 10$. The histogram and normal quantile plot in Figure 11 illustrate the performance of the normal approximation for $R_{n}^{(i)}$ implied by Theorem 5 . The p-value (0.60) for the Kolmogorov-Smirnov test also supports the hypothesis of standard normality for the residuals.

Example 3. Consider the sequence $X_{t}:=\exp \left(\theta_{0} Z_{t}+\theta_{1} Z_{t-1}\right)$, where $\theta_{0}=\sqrt{3} / 2, \theta_{1}=-1 / 2$, i.e., $\left\{X_{i}\right\}$ is a 1-dependent log-normal sequence, $X_{t} \stackrel{\mathrm{D}}{=} \exp \left(Z_{t}\right)$. In this case the quadratic entropy $h_{2}(\mathcal{P})=-\log \left(e^{1 / 4} /(2 \sqrt{\pi})\right)$. Figure 2 shows the accuracy of the normal approximation in Theorem 5 for the residuals $R_{n}^{(i)}:=\sqrt{n}\left(\tilde{Q}_{n}-q_{2}\right) / w_{r, n}, i=1, \ldots, N_{\text {sim }}$, where $n=500, r=4$, and $\epsilon=\epsilon_{0}=$ $3 / 100$. The histogram, normal quantile plot, and p-value (0.36) of the Kolmogorov-Smirnov test imply that the normality hypothesis can not be rejected.

Example 4. Estimation of the quadratic functional $q_{2}(\mathcal{P})$ for the 1-dependent Cauchy sequence $X_{t}=Z_{t} / Z_{t+1}$. Here $\mathcal{P}$ is the Cauchy distribution with $q_{2}(\mathcal{P})=1 /(2 \pi)$. We simulate residuals $R_{n}^{(i)}:=n \epsilon^{d / 2}\left(\tilde{Q}_{n}-q_{2}\right) / u_{n}, i=1, \ldots, N_{\text {sim }}$, where $n=500, \epsilon=1 / 100$, and $N_{\text {sim }}=500$. Figure 3 illustrates the performance of the normal approximation of $R_{n}^{(i)}$ indicated by Theorem 6 . The histogram, normal quantile plot, and p-value (0.47) for the Kolmogorov-Smirnov test allow to accept the hypothesis of standard normality. 
MA(2) sample

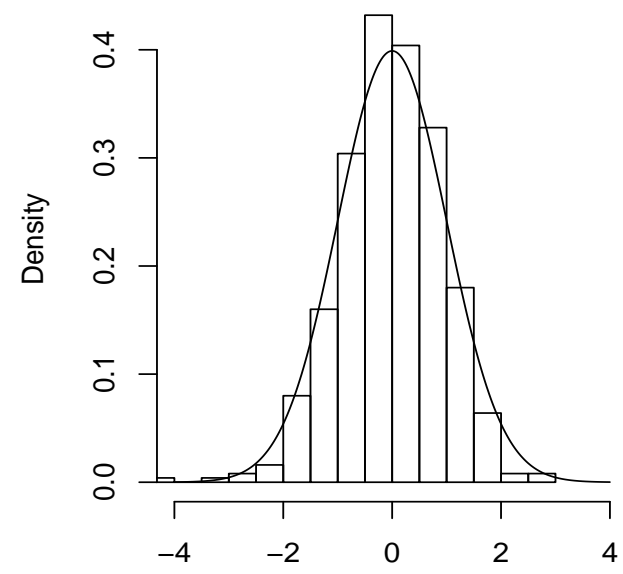

Normal quantile plot

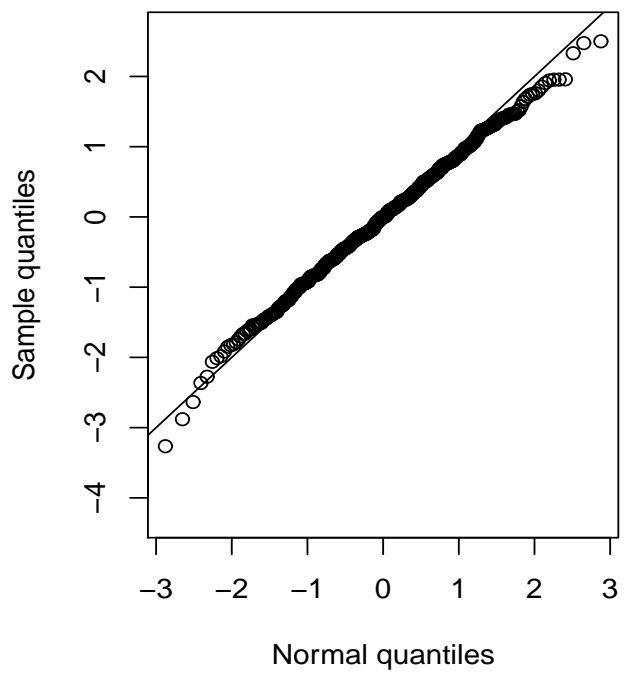

Figure 1: A 2-dependent MA(2) time series; sample size $n=500, r=6$, and $\epsilon=\epsilon_{0}=1 / 10$. Standard normal approximation for the normalized residuals; $N_{\text {sim }}=500$.
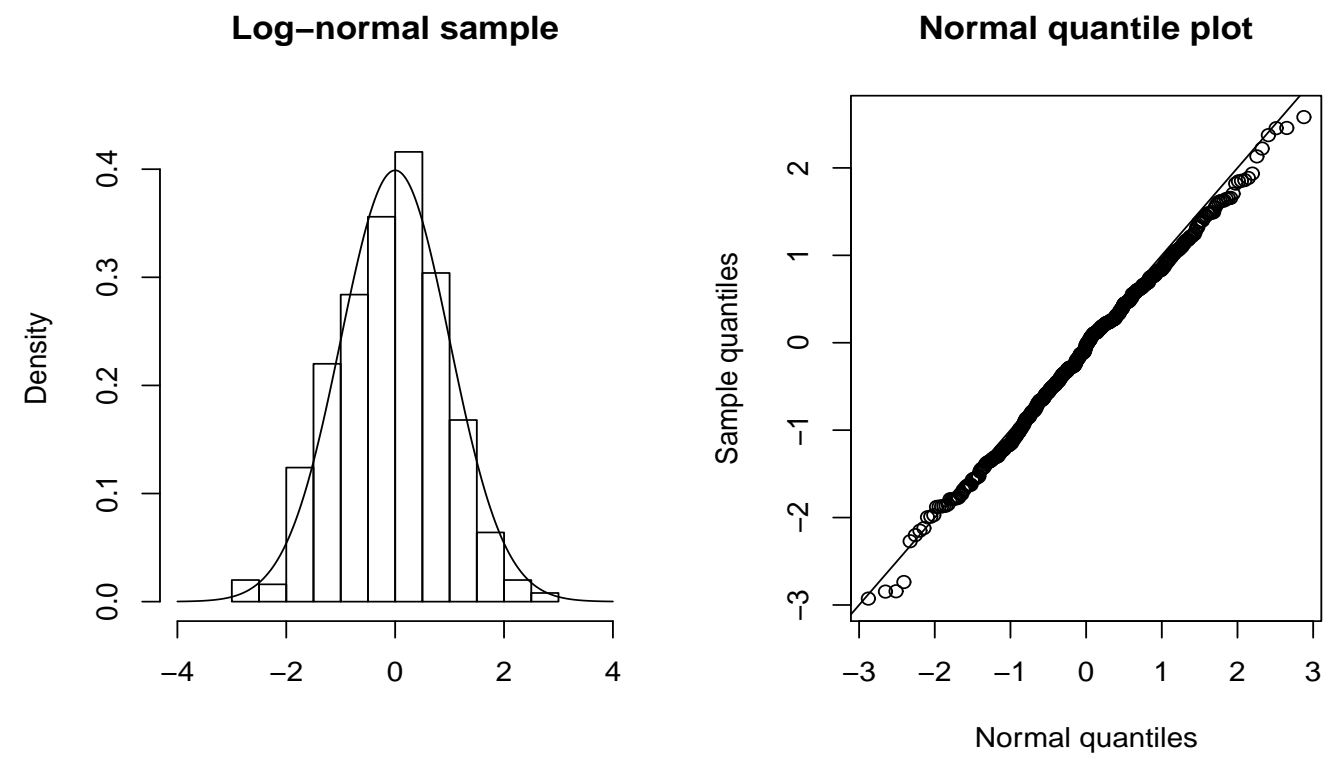

Figure 2: A 1-dependent log-normal sequence; sample size $n=500, r=4$, and $\epsilon=\epsilon_{0}=3 / 100$. Standard normal approximation for the normalized residuals; $N_{\text {sim }}=500$. 


\section{Cauchy sample}

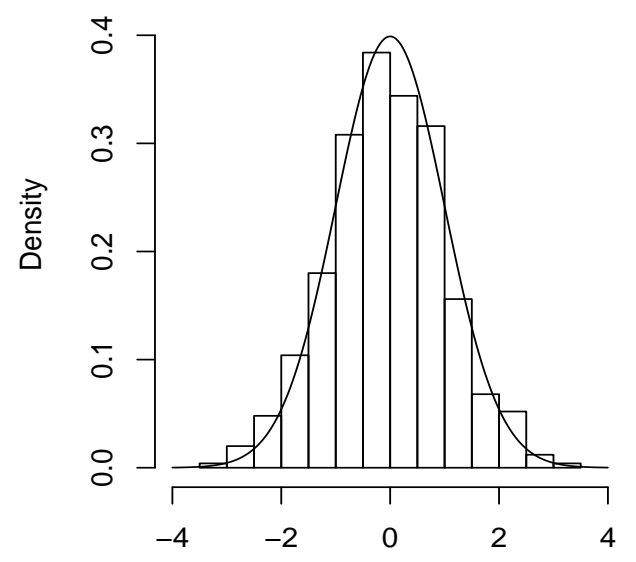

Normal quantile plot

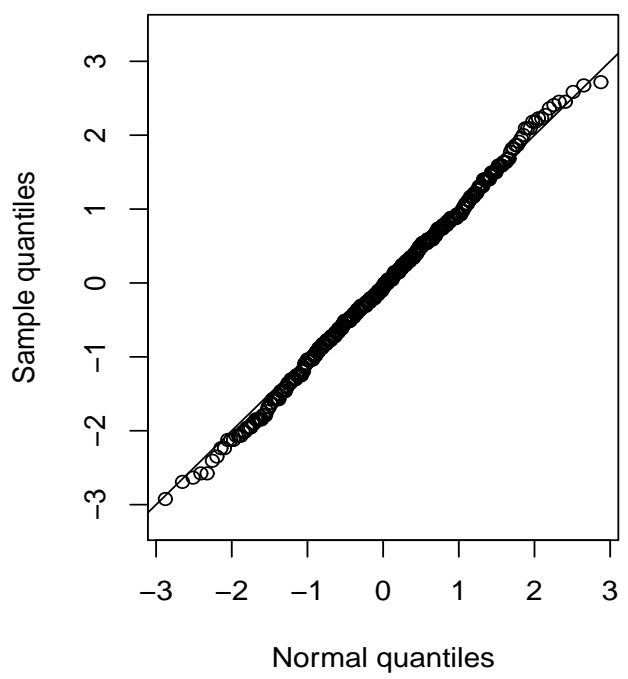

Figure 3: A 1-dependent Cauchy sequence; sample size $n=500$ and $\epsilon=1 / 100$. Standard normal approximation for the normalized residuals; $N_{\text {sim }}=500$.

\section{Applications}

\section{$\epsilon$-Keys in time series databases}

Let a time series database $T$ be a matrix with $n$ random records (or tuples) $r_{U}(j), j=1, \ldots, n$, and $k$ attributes, $U=\{1, \ldots, k\}$, with continuous tuple distribution with density $p(x)=p_{U}(x), x \in$ $R^{k}$. As contrast to conventional static databases, the ordering of records in $T$ is significant, i.e., the timestamp $j$ can be associated with an additional attribute for $r_{U}(j)$. For example, time series databases are used for modelling stock market, environmental (e.g., weather) or web usage data (see, e.g., Last et al., 2001). Then the database $T$ can be considered as a sample from a vector time series $\left\{r_{U}(t)\right\}_{t=1}^{\infty}$. Assume additionally that $\left\{r_{U}(t)\right\}$ is a stationary $m$-dependent time series. A subset $A \subseteq U,|A|=d \leq k$, is called an $\epsilon$-key if $N_{n}(A)=0$, i.e., there are no $\epsilon$-close in attributes $A$ sub-records $r_{A}(j), j=1, \ldots, n$. The distribution of $N_{n}(A)$ characterizes the capability of $A$ to distinguish records in $T$ and can be used to measure the complexity of a database design for further optimization, e.g., for optimal $\epsilon$-key selection or searching dependencies between attributes (or association rules) (see, e.g., Thalheim, 2000, Seleznjev and Thalheim, 2008, Leonenko and Seleznjev, 2010). Now Theorem 1(ii) gives an approximation of the probability that $A$ is an $\epsilon$-key, $P\left\{N_{n}(A)=0\right\} \sim e^{-\mu_{n}}$, where

$$
\mu_{n}=\frac{n(n-1)}{2} \epsilon^{d} b_{1}(d) \tilde{q}_{2, \epsilon}+\mathrm{o}\left(n \epsilon^{d / 2}\right) \sim \frac{1}{2} a b_{1}(d) q_{2} \text { as } n \rightarrow \infty, a>0,
$$

i.e., asymptotically optimal $\epsilon$-key candidates are amongst $A,|A|=d$, sets with minimal value of the quadratic functional $q_{2}$ and the corresponding estimators of $q_{2}$ are applicable with various asymptotics for $\epsilon$ and $n$ (Remark 2 and Theorems 2, 4, 5, and 6). 


\section{Entropy maximizing distributions for stationary m-dependent sequences}

Note that conditions of consistency for our estimate of the quadratic Rényi entropy $h_{2}(\mathcal{P}$ ) (see Theorem 2(ii)) are rather weak and can be easily verified for many statistical models. Hence, one can use these consistent estimators to build goodness of fit tests based on the maximum entropy principle, see, e.g., Goria et al. (2005) and Leonenko and Seleznjev (2010), where similar approaches were proposed for Shannon and Rényi entropies, respectively. Let us remind some known facts about the maximum entropy principle, see, e.g., Johnson and Vignat (2007). Consider the following maximization problem: given a symmetric positive definite matrix $\Sigma>0$, for all densities $p(x)$ with mean $\mu$ and such that

$$
\int_{\Omega_{0}} p(x)(x-\mu)(x-\mu)^{T} d x=\Sigma, \quad \Omega_{0}:=\left\{x \in R^{d}:(x-\mu)^{T} \Sigma^{-1}(x-\mu) \leq 4+d\right\},
$$

the quadratic Rényi entropy $h_{2}(\mathcal{P})$ is uniquely maximized by the distribution $\mathcal{P}^{*}$ with density

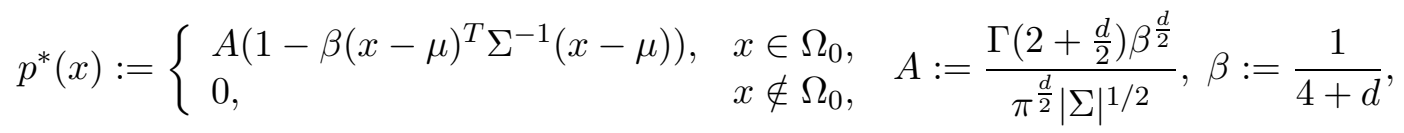

that is for all other densities support $\Omega_{0}$, mean $\mu$, and covariance matrix $\Sigma$, see (5), we have $h_{2}(\mathcal{P}) \leq h_{2}\left(\mathcal{P}^{*}\right)$, with equality if and only if $p=p^{*}$ almost everywhere with respect to the Lebesgue measure in $R^{d}$. The distribution $\mathcal{P}^{*}$ belongs to the class of multivariate Pearson type II distributions (or Student-r distributions) and its quadratic Rényi entropy

$$
h_{2}\left(\mathcal{P}^{*}\right)=-\log \frac{2 \Gamma\left(2+\frac{d}{2}\right)^{2} \beta^{\frac{d}{2}}}{\Gamma\left(3+\frac{d}{2}\right) \pi^{\frac{d}{2}}|\Sigma|^{1 / 2}} .
$$

For an i.i.d. sample, the goodness of fit test based on the maximum quadratic Rényi entropy principle was proposed by Leonenko and Seleznjev (2010). To generalize this test for $m$-dependent data, we need to show that there exists a stationary $m$-dependent sequence with marginal distribution (6). For one-dimensional processes, one can apply some results from Joe (1997). Henceforth, we use some definitions and notation from this book. It is known that for continuous multivariate distributions, the univariate marginals and the multivariate or dependent structures can be separated by copula. Let $C(u, v)$ be a bivariate copula with conditional distribution $C_{(2 \mid 1)}(v \mid u)=\partial C(u, v) / \partial u$. The inverse conditional distribution is denoted by $C_{(2 \mid 1)}^{-1}(s \mid u)$. Let $F$ be a continuous univariate distribution function and let $\left\{U_{i}\right\}$ be a sequence i.i.d. uniformly distributed $U(0,1)$-random variables. A 1-dependent sequence with marginal distribution $F$ is $Y_{t}=h\left(U_{t}, U_{t+1}\right)$, where $h(u, v)=F^{-1}\left[C_{(2 \mid 1)}^{-1}(v \mid u)\right]$. The marginal distribution of $Y_{t}$ is $F(y)$, see Joe (1997), p. 253, and the joint distribution of $\left(Y_{t}, Y_{t+1}\right)$ is of the form

$$
P\left(Y_{t} \leq x, Y_{t+1} \leq y\right)=\int_{0}^{1} C\left(C_{(2 \mid 1)}(F(x) \mid u), F(y)\right) d u
$$

In the same spirit, one can construct a stationary $m$-dependent sequence with given marginal distribution for any $m \geq 1$, see again Joe (1997), p. 255. In particular, for any $m \geq 1$, there exists a one-dimensional stationary $m$-dependent sequence with marginal distribution $F$, which has density (6) for $d=1$. For $d \geq 1$, the above copula construction seems difficult, but one can use 
again the results from Johnson and Vignat (2007), reformulated for quadratic entropy as follows: if $\left\{Z_{i}\right\}$ is a sequence of i.i.d. $N(0,1)$-variables, then

$$
X:=\frac{\sqrt{(d+4)} \Sigma^{1 / 2} \cdot Z}{\sqrt{\|Z\|^{2}+Z_{d+1}^{2}+\ldots+Z_{d+4}^{2}}}=f\left(Z_{1}, \ldots, Z_{d+4}\right),
$$

has density (6), where $Z:=\left(Z_{1}, \ldots, Z_{d}\right)^{T}$. Now a stationary $m$-dependent sequence with the marginal density (6) can be defined for all $m \in\{1, \ldots, d+3\}$, e.g., $X_{t}:=f\left(Z_{t}, \ldots, Z_{t+d+3}\right), t \geq$ $1, m=d+3$. Consequently, for any fixed $d$ and $m \in\{1, \ldots, d+3\}$, there exists a stationary $m$-dependent vector sequence with marginal maximum quadratic entropy distribution (6) $)$. Next, from (7) we get

$$
\frac{e^{h_{2}\left(\mathcal{P}^{*}\right)}}{|\Sigma|^{1 / 2}}=K_{d}:=\frac{\Gamma\left(3+\frac{d}{2}\right) \pi^{\frac{d}{2}}}{2 \Gamma\left(2+\frac{d}{2}\right)^{2} \beta^{\frac{d}{2}}} .
$$

Let $\mathcal{K}$ be a class of $d$-dimensional density functions $p(x), x \in R^{d}$, with support $\operatorname{supp}\{p\}=\Omega_{0}$, which satisfy condition (5). Note that the density $p^{*}(x)$ belongs to this class. Let $X_{1}, \ldots, X_{n}, n \geq 2$, be an $m$-dependent sample from a member of $\mathcal{K}$. Consider $\Sigma_{n}:=1 /(n-1) \sum_{i=1}^{n}\left(X_{i}-\bar{X}_{n}\right)\left(X_{i}-\right.$ $\left.\bar{X}_{n}\right)^{T}$, the sample covariance matrix, as a consistent estimate of $\Sigma$ as $n \rightarrow \infty$, where the sample mean $\bar{X}_{n}:=1 / n \sum_{i=1}^{n} X_{i}$. The consistency of the sample covariance matrix is a simple consequence of the asymptotic properties of stationary $m$-dependent processes, see, e.g., Lee, (1990), Anderson, (1994). Then under the null hypothesis $H_{0}: X_{1}, \ldots X_{n}$ is a sample from $m$-dependent data with density $p^{*}(x)$, we obtain from the Slutsky theorem and Theorem 2 (ii), that

$$
\left|\Sigma_{n}\right|^{-1 / 2} \exp \left\{\tilde{H}_{n}\right\} \stackrel{\mathrm{P}}{\rightarrow} K_{d} \text { as } n \rightarrow \infty,
$$

where $K_{d}$ is defined in (8), and $\tilde{H}_{n}$ is the consistent estimator of the quadratic Rényi entropy. Under the alternative $H_{1}: X_{1}, \ldots, X_{n}$ is a sample from any other member $p$ of $\mathcal{K}$, we find that

$$
\left|\Sigma_{n}\right|^{-1 / 2} \exp \left\{\tilde{H}_{n}\right\} \stackrel{\mathrm{P}}{\rightarrow} \frac{e^{h_{2}(\mathcal{P})}}{|\Sigma|^{1 / 2}}<K_{d} \text { as } n \rightarrow \infty .
$$

In other words, the above mentioned test is consistent against such alternatives. Note that $K_{1}=$ $\frac{5}{3} \sqrt{5} \simeq 3.727, K_{2}=\frac{9}{2} \pi \simeq 14.137, K_{3}=\frac{98}{15} \pi \sqrt{7} \simeq 54.304$.

\section{Proofs}

The following lemmas are used in the subsequent proofs.

Lemma 1 (Ch. 2, Lee, 1990) Let $k \geq 2$. Then the number of $k$-tuples of integers $1 \leq i_{1}<\cdots<$ $i_{k} \leq n$ that satisfy $i_{j}-i_{j-1}>h$ for $j=2,3, \ldots, k$ is $\left(\begin{array}{c}n-(k-1) h \\ k\end{array}\right)$.

Lemma 2 (Ch. 5, Billingsley, 1995) If, for each $k, Z_{n}^{(k)} \stackrel{\mathrm{D}}{\rightarrow} Z^{(k)}$ as $n \rightarrow \infty$, if $Z^{(k)} \stackrel{\mathrm{D}}{\rightarrow} Z$ as $k \rightarrow \infty$, and if

$$
\lim _{k \rightarrow \infty} \limsup _{n \rightarrow \infty} P\left(\left|Z_{n}^{(k)}-Z_{n}\right| \geq \delta\right)=0
$$

for positive $\delta$, then $Z_{n} \stackrel{\mathrm{D}}{\rightarrow} Z$. 


\section{Lemma 3}

(i) Assume that $p(x) \in L_{3}\left(R^{d}\right)$ and let $\tilde{p}_{X, \epsilon}(x):=b_{\epsilon}(d)^{-1} p_{X, \epsilon}(x)$. Then, for $h=0,1, \ldots$,

$$
\mathrm{E} \tilde{p}_{X, \epsilon}(X)=\tilde{q}_{2, \epsilon} \rightarrow q_{2} \quad \text { and } \quad \mathrm{E} \tilde{p}_{X, \epsilon}\left(X_{1}\right) \tilde{p}_{X, \epsilon}\left(X_{1+h}\right) \rightarrow \mathrm{E} p\left(X_{1}\right) p\left(X_{1+h}\right) \text { as } \epsilon \rightarrow 0
$$

and thus $b_{\epsilon}(d)^{-2} \sigma_{1, h, \epsilon}^{2} \rightarrow \operatorname{Cov}\left(p\left(X_{1}\right), p\left(X_{1+h}\right)\right)$ as $\epsilon \rightarrow 0$.

(ii) If $\mathcal{A}$ is satisfied, then

$$
\sup _{i \neq j} P\left(d\left(X_{i}, X_{j}\right) \leq \epsilon\right)=\mathrm{o}\left(\epsilon^{d / 2}\right) \text { as } \epsilon \rightarrow 0
$$

(iii) If $\mathcal{A}$ is satisfied, then

$$
\sup _{\left\{i_{1}, i_{2}\right\} \neq\left\{j_{1}, j_{2}\right\}} P\left(d\left(X_{i_{1}}, X_{j_{1}}\right) \leq \epsilon, d\left(X_{i_{2}}, X_{j_{2}}\right) \leq \epsilon\right)=\mathrm{o}\left(\epsilon^{d}\right) \text { as } \epsilon \rightarrow 0 .
$$

Proof. (i) We use the following result from Lemma 1 in Källberg and Seleznjev (2012): for random vectors $X$ and $Y$ with densities $p_{X}(x), p_{Y}(x) \in L_{a+1}\left(R^{d}\right), x \in R^{d}, a \geq 0$,

$$
\mathrm{E} \tilde{p}_{X, \epsilon}(Y)^{a} \rightarrow \mathrm{E} p(Y)^{a} \text { as } \epsilon \rightarrow 0 .
$$

Note that (9) immediately implies $\tilde{q}_{2, \epsilon} \rightarrow q_{2}$. Furthermore, if $Y$ is defined to have density $p_{Y}(x):=$ $p(x)^{2} / q_{2}, x \in R^{d}$, we obtain from (9) that

$$
\mathrm{E} \tilde{p}_{X, \epsilon}\left(X_{1}\right) p\left(X_{1}\right)=q_{2} \mathrm{E} \tilde{p}_{X, \epsilon}(Y) \rightarrow q_{2} \mathrm{E} p(Y)=\mathrm{E} p\left(X_{1}\right)^{2} \text { as } \epsilon \rightarrow 0 .
$$

Now consider the decomposition

$$
\begin{aligned}
\mathrm{E} \tilde{p}_{X, \epsilon}\left(X_{1}\right) \tilde{p}_{X, \epsilon}\left(X_{1+h}\right)= & \mathrm{E} p\left(X_{1}\right) p\left(X_{1+h}\right)+\mathrm{E}\left(\tilde{p}_{X, \epsilon}\left(X_{1}\right)-p\left(X_{1}\right)\right) \tilde{p}_{X, \epsilon}\left(X_{1+h}\right) \\
& +\mathrm{E}\left(\left(\tilde{p}_{X, \epsilon}\left(X_{1+h}\right)-p\left(X_{1+h}\right)\right) p\left(X_{1}\right) .\right.
\end{aligned}
$$

By the stationarity of $\left\{X_{i}\right\}$ and Hölder's inequality, the last two terms in (11) are in absolute value bounded by

$$
\left(\mathrm{E}\left(\tilde{p}_{X, \epsilon}\left(X_{1}\right)-p\left(X_{1}\right)\right)^{2}\right)^{1 / 2}\left(\mathrm{E} \tilde{p}_{X, \epsilon}\left(X_{1}\right)^{2}\right)^{1 / 2}
$$

which by (9) and (10) tends to zero as $\epsilon \rightarrow 0$. Hence, $\mathrm{E} \tilde{p}_{X, \epsilon}\left(X_{1}\right) \tilde{p}_{X, \epsilon}\left(X_{1+h}\right) \rightarrow \operatorname{E} p\left(X_{1}\right) p\left(X_{1+h}\right)$ and the assertion follows.

(ii) First note that the indices $t_{3}$ and $t_{4}$ can be chosen in such a way that $X_{t_{3}}$ and $X_{t_{4}}$ are independent and also independent of $\left\{X_{t_{1}}, X_{t_{2}}\right\}$. For the corresponding density of $\left(X_{t_{1}}, X_{t_{2}}, X_{t_{3}}, X_{t_{4}}\right)$, we have $p_{\mathbf{t}}\left(x_{1}, x_{2}, x_{3}, x_{4}\right)=p_{t_{1}, t_{2}}\left(x_{1}, x_{2}\right) p\left(x_{3}\right) p\left(x_{4}\right)$, where $p_{t_{1}, t_{2}}\left(x_{1}, x_{2}\right)$ is the density of $\left(X_{t_{1}}, X_{t_{2}}\right)$. Consequently, from assumption $\mathcal{A}$,

$$
\begin{aligned}
& g_{\mathbf{t}}\left(x_{1}, x_{4}\right) \in L_{1}\left(R^{2 d}\right), \\
& g_{\mathbf{t}}\left(x_{1}, x_{4}\right):=\left(\int_{R^{d}} p_{t_{1}, t_{2}}\left(x_{1}, x_{2}\right)^{2} p\left(x_{3}\right)^{2} p\left(x_{4}\right)^{2} d x_{2} d x_{3}\right)^{1 / 2}=p\left(x_{4}\right) q_{2}^{1 / 2}\left(\int_{R^{d}} p_{t_{1}, t_{2}}\left(x_{1}, x_{2}\right)^{2} d x_{2}\right)^{1 / 2} .
\end{aligned}
$$


Integrating $g_{\mathbf{t}}\left(x_{1}, x_{4}\right)$ with respect to $x_{4}$ gives

$$
g_{t_{1}, t_{2}}\left(x_{1}\right) \in L_{1}\left(R^{d}\right), \quad g_{t_{1}, t_{2}}\left(x_{1}\right):=\left(\int_{R^{d}} p_{t_{1}, t_{2}}\left(x_{1}, x_{2}\right)^{2} d x_{2}\right)^{1 / 2} .
$$

Therefore, by Hölder's inequality,

$$
\begin{aligned}
P\left(d\left(X_{t_{1}}, X_{t_{2}}\right) \leq \epsilon\right) & =\int_{\|x-y\| \leq \epsilon} p_{t_{1}, t_{2}}(x, y) d x d y=\int_{R^{d}}\left(\int_{\|x-y\| \leq \epsilon} p_{t_{1}, t_{2}}(x, y) d y\right) d x \\
& \leq \int_{R^{d}}\left(\int_{\|x-y\| \leq \epsilon} d y\right)^{1 / 2}\left(\int_{\|x-y\| \leq \epsilon} p_{t_{1}, t_{2}}(x, y)^{2} d y\right)^{1 / 2} d x \\
& =b_{\epsilon}(d)^{1 / 2} \int_{R^{d}} h_{t_{1}, t_{2}}^{(\epsilon)}(x) d x
\end{aligned}
$$

where

$$
h_{t_{1}, t_{2}}^{(\epsilon)}(x):=\left(\int_{\|x-y\| \leq \epsilon} p_{t_{1}, t_{2}}(x, y)^{2} d y\right)^{1 / 2} \leq\left(\int_{R^{d}} p_{t_{1}, t_{2}}(x, y)^{2} d y\right)^{1 / 2}=g_{t_{1}, t_{2}}(x) .
$$

Since $g_{t_{1}, t_{2}}(x) \in L_{1}\left(R^{d}\right)$ and $h_{t_{1}, t_{2}}^{(\epsilon)}(x) \rightarrow 0$ as $\epsilon \rightarrow 0$, the dominated convergence theorem yields $\int_{R^{d}} h_{t_{1}, t_{2}}^{(\epsilon)}(x) d x \rightarrow 0$ as $\epsilon \rightarrow 0$, and hence from (12) we obtain

$$
P\left(d\left(X_{t_{1}}, X_{t_{2}}\right) \leq \epsilon\right)=\mathrm{o}\left(\epsilon^{d / 2}\right) \text { as } \epsilon \rightarrow 0
$$

for each distinct pair $\left\{t_{1}, t_{2}\right\}$. Finally, the stationarity and $m$-dependence of the sequence $\left\{X_{i}\right\}$ imply that $P\left(d\left(X_{t_{1}}, X_{t_{2}}\right) \leq \epsilon\right)$ attains a finite number of values as $t_{1}$ and $t_{2}$ vary. Thus, the rate of convergence in (13) remains valid if we take the supremum over distinct $\left\{t_{1}, t_{2}\right\}$ as desired, so the statement follows.

(iii) Since the argument is similar to that of (ii), we show the main steps only. First assume that $i_{1}=i_{2}$ and $j_{1} \neq j_{2}$. Under $\mathcal{A}$, it can be shown in a similar way as above that for each 3 -tuple of distinct positive integers $\left(t_{1}, t_{2}, t_{3}\right)$, the density $p_{t_{1}, t_{2}, t_{3}}\left(x_{1}, x_{2}, x_{3}\right)$ of $\left(X_{t_{1}}, X_{t_{2}}, X_{t_{3}}\right)$ satisfies

$$
g_{t_{1}, t_{2}, t_{3}}\left(x_{1}\right) \in L_{1}\left(R^{d}\right), \quad g_{t_{1}, t_{2}, t_{3}}\left(x_{1}\right):=\left(\int_{R^{d}} p_{t_{1}, t_{2}, t_{3}}\left(x_{1}, x_{2}, x_{3}\right)^{2} d x_{2} d x_{3}\right)^{1 / 2} .
$$

Now introduce the random vectors $Y_{i}:=\left(X_{i_{1}}, X_{i_{1}}\right)$ and $Y_{j}:=\left(X_{j_{1}}, X_{j_{2}}\right)$ in $R^{2 d}$ and note that

$$
P\left(d\left(X_{i_{1}}, X_{j_{1}}\right) \leq \epsilon, d\left(X_{i_{1}}, X_{j_{2}}\right) \leq \epsilon\right) \leq P\left(\left\|Y_{i}-Y_{j}\right\|_{2 d} \leq 2 \epsilon\right),
$$

where $\|\cdot\|_{2 d}$ is the Euclidean norm in $R^{2 d}$. Define the coordinate vectors $y_{1}:=\left(x_{1}, x_{1}\right)$ and $y_{2}:=\left(x_{2}, x_{3}\right)$. By Hölder's inequality,

$$
\begin{aligned}
P\left(\left\|Y_{i}-Y_{j}\right\|_{2 d} \leq 2 \epsilon\right) & =\int_{\left\|y_{1}-y_{2}\right\|_{2 d} \leq 2 \epsilon} p_{i_{1}, j_{1}, j_{2}}\left(x_{1}, x_{2}, x_{3}\right) d x_{1} d x_{2} d x_{3} \\
& \leq b_{2 \epsilon}(2 d)^{1 / 2} \int_{R^{d}} h_{i_{1}, j_{1}, j_{2}}^{(\epsilon)}\left(x_{1}\right) d x_{1},
\end{aligned}
$$


where

$$
h_{i_{1}, j_{1}, j_{2}}^{(\epsilon)}\left(x_{1}\right):=\left(\int_{\left\|y_{1}-y_{2}\right\|_{2 d} \leq 2 \epsilon} p_{i_{1}, j_{1}, j_{2}}\left(x_{1}, x_{2}, x_{3}\right)^{2} d x_{2} d x_{3}\right)^{1 / 2} \leq g_{i_{1}, j_{1}, j_{2}}\left(x_{1}\right) .
$$

By the dominated convergence theorem, $\int_{R^{d}} h_{i_{1}, j_{1}, j_{2}}^{(\epsilon)}\left(x_{1}\right) d x_{1} \rightarrow 0$ as $\epsilon \rightarrow 0$ and therefore, since $b_{2 \epsilon}(2 d)^{1 / 2}=C_{d} \epsilon^{d}, C_{d}>0$, we get from (14) and (15) that

$$
P\left(d\left(X_{i_{1}}, X_{j_{1}}\right) \leq \epsilon, d\left(X_{i_{2}}, X_{j_{2}}\right) \leq \epsilon\right)=\mathrm{o}\left(\epsilon^{d}\right) \text { as } \epsilon \rightarrow 0
$$

where $i_{1}=i_{2}$ and $j_{1} \neq j_{2}$. By a similar argument, this also valid when $i_{1} \neq i_{2}$ and $j_{1} \neq j_{2}$. Finally, from the stationarity and $m$-dependence of $\left\{X_{i}\right\}$, the rate of convergence in (16) still holds if we take the supremum over distinct pairs of pairs. This completes the proof.

Proof of Proposition 1, (i) Define the index set $\mathcal{I}=\mathcal{I}(n, m):=\{(i, j): 1 \leq i<j \leq n, j-i>m\}$ and the reduced form of $N_{n}$ :

$$
N_{n}^{*}:=\sum_{(i, j) \in \mathcal{I}} I\left(d\left(X_{i}, X_{j}\right) \leq \epsilon\right)
$$

Since $\operatorname{E} I\left(d\left(X_{i}, X_{j}\right) \leq \epsilon\right)=q_{2, \epsilon}$ when $(i, j) \in \mathcal{I}$, Lemmas 1 and 3 yield the claim for the expectation:

$$
\begin{aligned}
\mathrm{E}_{n} & =\mathrm{E}_{n}^{*}+\sum_{h=1}^{m}(n-h) P\left(d\left(X_{1}, X_{1+h}\right) \leq \epsilon\right)=\left(\begin{array}{c}
n-m \\
2
\end{array}\right) q_{2, \epsilon}+\mathrm{o}\left(n \epsilon^{d / 2}\right) \\
& =\left(\begin{array}{l}
n \\
2
\end{array}\right) q_{2, \epsilon}+\mathrm{o}\left(n \epsilon^{d / 2}\right) \text { as } n \rightarrow \infty .
\end{aligned}
$$

For the variance of $N_{n}$, we first study the variance of $N_{n}^{*}$. To this end, we need to consider $|\mathcal{I}|^{2}=\left(\begin{array}{c}n-m \\ 2\end{array}\right)^{2}$ terms of the form

$$
\operatorname{Cov}\left(I\left(d\left(X_{s_{1}}, X_{s_{2}}\right) \leq \epsilon\right), I\left(d\left(X_{t_{1}}, X_{t_{2}}\right) \leq \epsilon\right)\right)
$$

where $\left(s_{1}, s_{2}\right),\left(t_{1}, t_{2}\right) \in \mathcal{I}$. To count the various types of such terms, we use results from Ch. 2.4.1, Theorem 1, in Lee (1990). It should be noted that these results are stated for $U$-statistics based on random variables, rather than random vectors, but the argument is merely combinatorial and thus also valid here.

1) When $\left|s_{i}-t_{j}\right|>m, i, j=1,2$, the random variables in (18) are independent and the covariance is zero.

2) Only two random variables are involved, i.e., $s_{1}=t_{1}$ and $s_{2}=t_{2}$, so the covariance is $\operatorname{Var}\left(I\left(d\left(X_{s_{1}}, X_{s_{2}}\right)<\epsilon\right)\right)=q_{2, \epsilon}-q_{2, \epsilon}^{2}=q_{2, \epsilon}+\mathrm{O}\left(\epsilon^{2 d}\right)$ as $\epsilon \rightarrow 0$. The number of such terms is $|\mathcal{I}|=\left(\begin{array}{c}n-m \\ 2\end{array}\right)$.

3) Exactly one of the four possible differences $\left|s_{i}-t_{j}\right|$ is zero and the rest are greater than $m$. By conditioning, the covariance is $\operatorname{Cov}\left(I\left(d\left(X_{s_{1}}, X_{s_{2}}\right) \leq \epsilon\right), I\left(d\left(X_{s_{1}}, X_{t_{2}}\right) \leq \epsilon\right)\right)=\sigma_{1,0, \epsilon}^{2}$. There are $6\left(\begin{array}{c}n-2 m \\ 3\end{array}\right)$ terms of this type.

4) For $h=1, \ldots, m, 0<\left|s_{i}-t_{j}\right|=h \leq m$ for one of the differences $\left|s_{i}-t_{j}\right|$ and the others are greater than $m$. Then the covariance is $\operatorname{Cov}\left(I\left(d\left(X_{s_{1}}, X_{s_{2}}\right) \leq \epsilon\right), I\left(d\left(X_{s_{1}+h}, X_{t_{2}}\right) \leq \epsilon\right)\right)=$ $\sigma_{1, h, \epsilon}^{2}$. The number of terms of this type is $12\left(\begin{array}{c}n-2 m-h \\ 3\end{array}\right)$. 
5) The number of the remaining terms is $\mathrm{O}\left(n^{2}\right)$, so Lemma $3($ iii) indicates that their sum is $\mathrm{o}\left(n^{2} \epsilon^{d}\right)$ as $n \rightarrow \infty$.

From the above and Lemma 3, we obtain

$$
\begin{aligned}
\operatorname{Var}\left(N_{n}^{*}\right) & =\sum_{\left(s_{1}, s_{2}\right) \in \mathcal{I}} \sum_{\left(t_{1}, t_{2}\right) \in \mathcal{I}} \operatorname{Cov}\left(I\left(d\left(X_{s_{1}}, X_{s_{2}}\right) \leq \epsilon\right), I\left(d\left(X_{t_{1}}, X_{t_{2}}\right) \leq \epsilon\right)\right) \\
& =\left(\begin{array}{c}
n-m \\
2
\end{array}\right) q_{2, \epsilon}+6\left(\begin{array}{c}
n-2 m \\
3
\end{array}\right) \sigma_{1,0, \epsilon}^{2}+\sum_{h=1}^{m} 12\left(\begin{array}{c}
n-2 m-h \\
3
\end{array}\right) \sigma_{1, h, \epsilon}^{2}+\mathrm{o}\left(n^{2} \epsilon^{d}\right) \\
& =\frac{n^{2}}{2} q_{2, \epsilon}+n^{3}\left(\sigma_{1,0, \epsilon}^{2}+2 \sum_{h=1}^{m} \sigma_{1, h, \epsilon}^{2}\right)+\mathrm{o}\left(n^{2} \epsilon^{d}\right) \text { as } n \rightarrow \infty .
\end{aligned}
$$

Next it is verified that $\operatorname{Var}\left(N_{n}^{*}\right)$ and $\operatorname{Var}\left(N_{n}\right)$ are close. Note that

$$
\operatorname{Var}\left(N_{n}\right)=(n-1) C_{1, n}+\ldots+(n-m) C_{m, n}+\operatorname{Cov}\left(N_{n}, N_{n}^{*}\right),
$$

where $C_{h, n}:=\operatorname{Cov}\left(N_{n}, I\left(d\left(X_{1}, X_{1+h}\right) \leq \epsilon\right)\right), h=1, \ldots, m$. We have

$$
\begin{aligned}
C_{h, n} & =\sum_{1 \leq i<j \leq n} \operatorname{Cov}\left(I\left(d\left(X_{i}, X_{j}\right) \leq \epsilon\right), I\left(d\left(X_{1}, X_{1+h}\right) \leq \epsilon\right)\right. \\
& =\operatorname{Var}\left(I\left(d\left(X_{1}, X_{1+h}\right) \leq \epsilon\right)\right)+\sum_{\substack{1 \leq i<j \leq n \\
i, j\} \neq\{1,1+h\}}} \operatorname{Cov}\left(I\left(d\left(X_{i}, X_{j}\right) \leq \epsilon\right), I\left(d\left(X_{1}, X_{1+h}\right) \leq \epsilon\right)\right),
\end{aligned}
$$

where the number of pairs $(i, j)$ in the last sum for which $I\left(d\left(X_{i}, X_{j}\right) \leq \epsilon\right)$ and $I\left(d\left(X_{1}, X_{1+h}\right) \leq \epsilon\right)$ are not independent is $\mathrm{O}(n)$. From Lemma 3, we get $C_{h, n}=\mathrm{o}\left(\epsilon^{d / 2}\right)+\mathrm{o}\left(n \epsilon^{d}\right)$ as $n \rightarrow \infty$, and consequently (201) leads to

$$
\operatorname{Var}\left(N_{n}\right)=\operatorname{Cov}\left(N_{n}, N_{n}^{*}\right)+\mathrm{o}\left(n \epsilon^{d / 2}\right)+\mathrm{o}\left(n^{2} \epsilon^{d}\right) \text { as } n \rightarrow \infty .
$$

Furthermore, observe that

$$
\operatorname{Var}\left(N_{n}\right)=\operatorname{Var}\left(N_{n}^{*}\right)+\operatorname{Var}\left(N_{n}-N_{n}^{*}\right)+2 \operatorname{Cov}\left(N_{n}^{*}, N_{n}-N_{n}^{*}\right)
$$

and hence (21) gives

$$
\operatorname{Var}\left(N_{n}\right)=\operatorname{Var}\left(N_{n}^{*}\right)-\operatorname{Var}\left(N_{n}-N_{n}^{*}\right)+\mathrm{o}\left(n \epsilon^{d / 2}\right)+\mathrm{o}\left(n^{2} \epsilon^{d}\right) \text { as } n \rightarrow \infty .
$$

The next step is to show that

$$
\operatorname{Var}\left(N_{n}-N_{n}^{*}\right)=\mathrm{o}\left(n \epsilon^{d / 2}\right)+\mathrm{o}\left(n^{2} \epsilon^{d}\right) \text { as } n \rightarrow \infty .
$$

To this end, first note that Lemma 3 (ii) implies

$$
\mathrm{E}\left(N_{n}-N_{n}^{*}\right)=\sum_{\substack{1 \leq i<j \leq n \\ j-i \leq m}} \mathrm{E} I\left(d\left(X_{i}, X_{j}\right) \leq \epsilon\right)=\mathrm{o}\left(n \epsilon^{d / 2}\right) \text { as } n \rightarrow \infty,
$$

since the number of terms in this sum is $\mathrm{O}(n)$, so we only need to prove that

$$
\mathrm{E}\left(N_{n}-N_{n}^{*}\right)^{2}=\mathrm{o}\left(n \epsilon^{d / 2}\right)+\mathrm{o}\left(n^{2} \epsilon^{d}\right) \text { as } n \rightarrow \infty .
$$


We have

$$
\mathrm{E}\left(N_{n}-N_{n}^{*}\right)^{2}=\sum_{\substack{1 \leq i<j \leq n \\ j-i \leq m}} \sum_{\substack{1 \leq i^{\prime}<j^{\prime} \leq n \\ j^{\prime}-i^{\prime} \leq m}} \mathrm{E} I\left(d\left(X_{i}, X_{j}\right) \leq \epsilon\right) I\left(d\left(X_{i^{\prime}}, X_{j^{\prime}}\right) \leq \epsilon\right) .
$$

There are $\mathrm{O}\left(n^{2}\right)$ terms in this double sum. Moreover, $\mathrm{O}(n)$ of these have the form $P\left(d\left(X_{i}, X_{j}\right) \leq \epsilon\right)$ and the remaining are of the type $P\left(d\left(X_{i_{1}}, X_{j_{1}}\right) \leq \epsilon, d\left(X_{i_{2}}, X_{j_{2}}\right) \leq \epsilon\right)$, where $\left\{i_{1}, j_{1}\right\} \neq\left\{i_{2}, j_{2}\right\}$, so (25) follows from Lemma 3. Therefore (23) holds true, and combining with (22) and (19), we get

$$
\operatorname{Var}\left(N_{n}\right)=\frac{n^{2}}{2} q_{2, \epsilon}+n^{3}\left(\sigma_{1,0, \epsilon}^{2}+2 \sum_{h=1}^{m} \sigma_{1, h, \epsilon}^{2}\right)+\mathrm{o}\left(n \epsilon^{d / 2}\right)+\mathrm{o}\left(n^{2} \epsilon^{d}\right) \text { as } n \rightarrow \infty .
$$

The assertion is proved.

(ii) If $\zeta_{1, m}>0$, the claim follows directly from Lemma 3 and (26). When $\zeta_{1, m}=0$, we have $\sup _{n \geq 1}\left\{n \epsilon^{d}\right\}<\infty$ by assumption. Hence, from (26) and the condition $n^{2} \epsilon^{d} \rightarrow a, 0<a \leq \infty$,

$$
\frac{\operatorname{Var}\left(N_{n}\right)}{\frac{1}{2} b_{1}(d) q_{2} n^{2} \epsilon^{d}}=1+\mathrm{o}\left(n \epsilon^{d}\right)+\mathrm{o}\left(1 /\left(n \epsilon^{d / 2}\right)\right)+\mathrm{o}(1) \rightarrow 1 \text { as } n \rightarrow \infty .
$$

This completes the proof.

Proof of Theorem 1, (i) From the assumption $n^{2} \epsilon^{d} \rightarrow 0$ and Proposition 1(i), we get $\mu_{n}, \sigma_{n}^{2} \rightarrow 0$. Consequently, $N_{n} \stackrel{\mathrm{P}}{\rightarrow} 0$ and the assertion follows.

(ii) By the condition $n^{2} \epsilon^{d} \rightarrow a, 0<a<\infty$, we have $n \epsilon^{d} \rightarrow 0$ and thus Proposition 1(i) and Lemma 3(i) yield $\mu_{n} \rightarrow \mu$. Moreover, since (25) and $n^{2} \epsilon^{d} \rightarrow a, 0<a<\infty$, imply $\mathrm{E}\left(N_{n}^{*}-N_{n}\right)^{2} \rightarrow 0$, it is enough to verify the Poisson convergence for $N_{n}^{*}$. For this we apply the Stein-Chen Poisson approximation method (Barbour et al., 1992). To measure the deviation between two probability distributions $\mathcal{P}_{1}$ and $\mathcal{P}_{2}$ of integer-valued random variables, we use the total variation distance

$$
d_{T V}\left(\mathcal{P}_{1}, \mathcal{P}_{2}\right):=\sup \left\{\left|\mathcal{P}_{1}(C)-\mathcal{P}_{2}(C)\right|: C \subset\{0,1, \ldots\}\right\}=\frac{1}{2} \sum_{i \geq 0}\left|\mathcal{P}_{1}(i)-\mathcal{P}_{2}(i)\right| .
$$

Recall the definition $\mathcal{I}:=\{(i, j): 1 \leq i<j \leq n, j-i>m\}$ and let $\mathcal{I}_{(i, j)}:=\mathcal{I} \backslash\{(i, j)\}$. Further, using the notation $I_{\epsilon}(i, j):=I\left(d\left(X_{i}, X_{j}\right) \leq \epsilon\right)$, we define

$$
\mathcal{I}_{(i, j)}^{0}:=\left\{(k, l) \in \mathcal{I}_{(i, j)}: I_{\epsilon}(k, l) \text { and } I_{\epsilon}(i, j) \text { are dependent }\right\} .
$$

Since $\mathrm{E}_{\epsilon}(i, j)=q_{2, \epsilon}$ for all $(i, j) \in \mathcal{I}$, Corollary 2.C.5 in Barbour et al. (1992) yields

$$
\begin{aligned}
d_{T V}\left(\mathcal{L}\left(N_{n}^{*}\right),\right. & \left.\operatorname{Po}\left(\mu_{n}^{*}\right)\right) \\
\leq & \frac{1}{\mu_{n}^{*}}\left(\sum_{(i, j) \in \mathcal{I}} q_{2, \epsilon}^{2}+\sum_{(i, j) \in \mathcal{I}} \sum_{(k, l) \in \mathcal{I}_{(i, j)}^{0}} q_{2, \epsilon}^{2}+\sum_{(i, j) \in \mathcal{I}} \sum_{(k, l) \in \mathcal{I}_{(i, j)}^{0}} \mathrm{E} I_{\epsilon}(i, j) I_{\epsilon}(k, l)\right),
\end{aligned}
$$

where $\mathcal{L}\left(N_{n}^{*}\right)$ denotes the distribution of $N_{n}^{*}$ and $\mu_{n}^{*}:=\mathrm{E} N_{n}^{*}$. The $m$-dependence of $\left\{X_{i}\right\}$ gives that the number of terms in $\mathcal{I}_{(i, j)}^{0}$, for each $(i, j) \in \mathcal{I}$, has the bound

$$
\left|\mathcal{I}_{(i, j)}^{0}\right| \leq n(4 m+2)
$$


so for the first two sums in (27), Lemma 3 and the condition $n^{2} \epsilon^{d} \rightarrow a, 0<a<\infty$, lead to

$$
\sum_{(i, j) \in \mathcal{I}} q_{2, \epsilon}^{2} \leq \sum_{(i, j) \in \mathcal{I}} \sum_{(k, l) \in \mathcal{I}_{(i, j)}^{0}} q_{2, \epsilon}^{2} \leq\left(\begin{array}{c}
n-m \\
2
\end{array}\right) n(4 m+2) q_{2, \epsilon}^{2} \sim(2 m+1) b_{1}(d)^{2} q_{2}^{2} a^{2} n^{-1} \text { as } n \rightarrow \infty .
$$

For the last sum in (27), we see that the various types of terms can be counted in a similar way as the covariances in (19). With the notation $q_{3, h, \epsilon}:=\mathrm{E} p_{X, \epsilon}\left(X_{1}\right) p_{X, \epsilon}\left(X_{1+h}\right)$, it follows from $n^{2} \epsilon^{d} \rightarrow a, 0<a<\infty$, and Lemma 3 (i) that

$$
\begin{aligned}
& \sum_{(i, j) \in \mathcal{I}} \sum_{(k, l) \in \mathcal{I}_{(i, j)}^{0}} \mathrm{E} I_{\epsilon}(i, j) I_{\epsilon}(k, l)=6\left(\begin{array}{c}
n-2 m \\
3
\end{array}\right) q_{3,0, \epsilon}+\sum_{h=1}^{m} 12\left(\begin{array}{c}
n-2 m-h \\
3
\end{array}\right) q_{3, h, \epsilon}+\mathrm{o}\left(n^{2} \epsilon^{d}\right) \\
& =b_{1}(d)^{2} a^{2}\left(\mathrm{E} p\left(X_{1}\right)^{2}+2 \sum_{h=1}^{m} \mathrm{E} p\left(X_{1}\right) p\left(X_{1+h}\right)\right) n^{-1}+\mathrm{o}\left(n^{-1}\right)+\mathrm{o}(1) \rightarrow 0 \text { as } n \rightarrow \infty .
\end{aligned}
$$

Note also that

$$
\mu_{n}^{*}=\left(\begin{array}{c}
n-m \\
2
\end{array}\right) q_{2, \epsilon} \rightarrow \mu \text { as } n \rightarrow \infty .
$$

By combining (27), (28), (29), and (30),

$$
d_{T V}\left(\mathcal{L}\left(N_{n}^{*}\right), P o\left(\mu_{n}^{*}\right)\right) \rightarrow 0 \text { as } n \rightarrow \infty .
$$

Finally, if $Z_{n} \sim \operatorname{Po}\left(\mu_{n}^{*}\right)$ and $Z \sim P o(\mu)$, then (30) yields $Z_{n} \stackrel{\mathrm{D}}{\rightarrow} Z$, so from (31) we obtain

$$
P\left(N_{n}^{*}=k\right)-P(Z=k)=P\left(N_{n}^{*}=k\right)-P\left(Z_{n}=k\right)+P\left(Z_{n}=k\right)-P(Z=k) \rightarrow 0 \text { as } n \rightarrow \infty,
$$

for each $k=0,1, \ldots$, and thus $N_{n}^{*} \stackrel{\mathrm{D}}{\rightarrow} Z$. The statement follows.

(iii) The idea of the proof is to apply Lemma 2 to the reduced form $N_{n}^{*}$ of $N_{n}$. In fact, by (24), (25), and Proposition 1(ii), for the last two terms in the decomposition

$$
\frac{N_{n}-\mathrm{E} N_{n}}{\sigma_{n}}=\frac{N_{n}^{*}-\mathrm{E} N_{n}^{*}}{\sigma_{n}}+\frac{\mathrm{E}\left(N_{n}^{*}-N_{n}\right)}{\sigma_{n}}+\frac{N_{n}-N_{n}^{*}}{\sigma_{n}},
$$

we have $\mathrm{E}\left(N_{n}^{*}-N_{n}\right) / \sigma_{n} \rightarrow 0$ and $\left(N_{n}-N_{n}^{*}\right) / \sigma_{n} \rightarrow 0$ (in quadratic mean). Consequently, it is enough to show

$$
Z_{n}:=\frac{N_{n}^{*}-\mathrm{E} N_{n}^{*}}{\sigma_{n}} \stackrel{\mathrm{D}}{\rightarrow} N(0,1) \text { as } n \rightarrow \infty .
$$

The weak convergence (32) follows from Lemma 2 if there exist successive approximations $\left\{Z_{n}^{(k)}\right\}_{k=1}^{\infty}$ of $Z_{n}$ such that

c1) $Z_{n}^{(k)} \stackrel{\mathrm{D}}{\rightarrow} Z^{(k)}$ as $n \rightarrow \infty$ and $Z^{(k)} \stackrel{\mathrm{D}}{\rightarrow} N(0,1)$ as $k \rightarrow \infty$.

c2) For every $\delta>0$,

$$
\lim _{k \rightarrow \infty} \limsup _{n \rightarrow \infty} P\left(\left|Z_{n}-Z_{n}^{(k)}\right| \geq \delta\right)=0
$$


In order to construct such $Z_{n}^{(k)}$, for each $k=1,2, \ldots$, define an integer $s=s(k, n):=[n /(k+m)]$ and consider the set of $k$-subsets of $\{1, \ldots, n\}$

$$
S_{i}^{(k)}:=\{(i-1)(k+m)+1, \ldots,(i-1)(k+m)+k\}, \quad i=1, \ldots, s .
$$

Now, based on the subset $\mathcal{I}^{(k)}:=\left\{(i, j): i \in S_{l}^{(k)}, j \in S_{t}^{(k)}, 1 \leq l<t \leq s\right\}$ of $\mathcal{I}$, let

$$
Z_{n}^{(k)}:=\frac{U_{n}^{(k)}-\mathrm{E} U_{n}^{(k)}}{\sigma_{n}}, \quad U_{n}^{(k)}=U_{n, \epsilon}^{(k)}:=\sum_{(i, j) \in \mathcal{I}^{(k)}} I\left(d\left(X_{i}, X_{j}\right) \leq \epsilon\right) .
$$

First we prove c2). Denote by $M_{2}, M_{3}$, and $\left\{M_{4, h}\right\}_{h=1}^{m}$, the numbers of terms of types 2,3 , and 4, respectively, defined in Proposition 1 $1(i)$. Furthermore, let $M_{2}^{(k)}, M_{3}^{(k)}$, and $\left\{M_{4, h}^{(k)}\right\}_{h=1}^{m}$ be the numbers of these terms that also appear in

$$
\operatorname{Var}\left(U_{n}^{(k)}\right)=\sum_{\left(s_{1}, s_{2}\right) \in \mathcal{I}^{(k)}} \sum_{\left(t_{1}, t_{2}\right) \in \mathcal{I}^{(k)}} \operatorname{Cov}\left(I\left(d\left(X_{s_{1}}, X_{s_{2}}\right) \leq \epsilon\right), I\left(d\left(X_{t_{1}}, X_{t_{2}}\right) \leq \epsilon\right)\right) .
$$

Since $\mathcal{I}^{(k)} \subseteq \mathcal{I}$, we observe that

$$
\begin{aligned}
\operatorname{Var}\left(N_{n}^{*}-U_{n}^{(k)}\right) \leq & \sum_{\left(s_{1}, s_{2}\right) \in \mathcal{I}} \sum_{\left(t_{1}, t_{2}\right) \in \mathcal{I}}\left|\operatorname{Cov}\left(I\left(d\left(X_{s_{1}}, X_{s_{2}}\right) \leq \epsilon\right), I\left(d\left(X_{t_{1}}, X_{t_{2}}\right) \leq \epsilon\right)\right)\right| \\
& -\sum_{\left(s_{1}, s_{2}\right) \in \mathcal{I}^{(k)}} \sum_{\left(t_{1}, t_{2}\right) \in \mathcal{I}^{(k)}}\left|\operatorname{Cov}\left(I\left(d\left(X_{s_{1}}, X_{s_{2}}\right) \leq \epsilon\right), I\left(d\left(X_{t_{1}}, X_{t_{2}}\right) \leq \epsilon\right)\right)\right| \\
= & \left(M_{2}-M_{2}^{(k)}\right) q_{2, \epsilon}+\left(M_{3}-M_{3}^{(k)}\right) \sigma_{1,0, \epsilon}^{2}+\sum_{h=1}^{m}\left(M_{4, h}-M_{4, h}^{(k)}\right)\left|\sigma_{1, h, \epsilon}^{2}\right| \\
& +\mathrm{o}\left(n^{2} \epsilon^{d}\right) \text { as } n \rightarrow \infty .
\end{aligned}
$$

First, for $h=1, \ldots, m$, we obtain a lower bound for $M_{4, h}^{(k)}$. Assume without loss of generality that $k \geq 2 m+1$. There are $\left(\begin{array}{l}s \\ 2\end{array}\right)(k-2 h)^{2}$ elements $\left(s_{1}, s_{2}\right)$ in $\mathcal{I}^{(k)}$ that also satisfy $\left(s_{1}+d_{1}, s_{2}+d_{2}\right) \in \mathcal{I}^{(k)}$, where $d_{1}, d_{2}= \pm h$. For every element of this type, an index $t_{1}$ with $\left|s_{1}-t_{1}\right|=h$ or $\left|s_{2}-t_{1}\right|=h$ can be chosen in 4 different ways. Moreover, for each such alternative we can choose $t_{2}$ in at least $(s-2) k$ different ways. We get that $M_{4, h}^{(k)}$ is bounded from below by

$$
\bar{M}_{4, h}^{(k)}:=\left(\begin{array}{l}
s \\
2
\end{array}\right)(k-2 h)^{2} 4(s-2) k \sim 2 \frac{(k-2 h)^{2} k}{(k+m)^{3}} n^{3} \text { as } n \rightarrow \infty .
$$

Further, by Proposition 1(ii), Lemma 3(i), and the limits $M_{4, h} \sim 2 n^{3}$ and (34), we get

$$
\begin{aligned}
\frac{\left(M_{4, h}-\bar{M}_{4, h}^{(k)}\right)\left|\sigma_{1, h, \epsilon}^{2}\right|}{\sigma_{n}^{2}} & =\frac{n^{3} \epsilon^{2 d}}{\sigma_{n}^{2}} \frac{\left(M_{4, h}-\bar{M}_{4, h}^{(k)}\right)\left|\sigma_{1, h, \epsilon}^{2}\right|}{n^{3} \epsilon^{2 d}} \\
& \rightarrow C_{4, h}\left(1-\frac{(k-2 h)^{2} k}{(k+m)^{3}}\right)\left|\operatorname{Cov}\left(p\left(X_{1}\right), p\left(X_{1+h}\right)\right)\right| \text { as } n \rightarrow \infty,
\end{aligned}
$$

for some $0 \leq C_{4, h}<\infty$. By a similar argument, we have lower bounds $\bar{M}_{2}^{(k)}$ and $\bar{M}_{3}^{(k)}$ for $M_{2}^{(k)}$ and $M_{3}^{(k)}$, respectively, such that, for some $0 \leq C_{2}<0$ and $0 \leq C_{3}<\infty$,

$$
\begin{gathered}
\frac{\left(M_{2}-\bar{M}_{2}^{(k)}\right) q_{2, \epsilon}}{\sigma_{n}^{2}}=\frac{n^{2} \epsilon^{d}}{\sigma_{n}^{2}} \frac{\left(M_{2}-\bar{M}_{2}^{(k)}\right) q_{2, \epsilon}}{n^{2} \epsilon^{d}} \rightarrow C_{2}\left(1-\frac{k^{2}}{(k+m)^{2}}\right) q_{2}, \\
\frac{\left(M_{3}-\bar{M}_{3}^{(k)}\right) \sigma_{1,0, \epsilon}^{2}}{\sigma_{n}^{2}}=\frac{n^{3} \epsilon^{2 d}}{\sigma_{n}^{2}} \frac{\left(M_{3}-\bar{M}_{3}^{(k)}\right) \sigma_{1,0, \epsilon}^{2}}{n^{3} \epsilon^{2 d}} \rightarrow C_{3}\left(1-\frac{k^{3}}{(k+m)^{3}}\right) \operatorname{Var}\left(p\left(X_{1}\right)\right) \text { as } n \rightarrow \infty .
\end{gathered}
$$


Now, from (33), (35), (36), and Proposition 1(ii),

$$
\lim _{k \rightarrow \infty} \lim _{n \rightarrow \infty} \operatorname{Var}\left(Z_{n}-Z_{n}^{(k)}\right)=\lim _{k \rightarrow \infty} \lim _{n \rightarrow \infty} \operatorname{Var}\left(\frac{N_{n}^{*}-U_{n}^{(k)}}{\sigma_{n}}\right)=0
$$

and hence, since $Z_{n}-Z_{n}^{(k)}$ has zero mean, c2) is implied by Chebyshev's inequality.

Next we prove that $\mathrm{c} 1)$. Let

$$
V(k):=\left\{\begin{array}{lr}
\frac{\frac{k^{2}}{2} q_{2}+\frac{k^{3}}{(k+m)^{2}} b_{1}(d) \zeta_{1, m}^{(k)} a}{\frac{1}{2} q_{2}+b_{1}(d) \zeta_{1, m} a}, & 0 \leq a<\infty, \\
\frac{k^{3}}{(k+m)^{3}} \frac{\zeta_{1, m}^{(k)}}{\zeta_{1, m}}, & a=\infty,
\end{array}\right.
$$

where

$$
\zeta_{1, m}^{(k)}:=\operatorname{Var}\left(p\left(X_{1}\right)\right)+2 \sum_{h=1}^{m}(1-h / k) \operatorname{Cov}\left(p\left(X_{1}\right), p\left(X_{1+h}\right)\right) .
$$

Note that, since $\zeta_{1, m}^{(k)} \rightarrow \zeta_{1, m}$ as $k \rightarrow \infty$, we have $V(k) \rightarrow 1$ as $k \rightarrow \infty$, so c1) follows if it can be verified that

$$
Z_{n}^{(k)} \rightarrow N(0, V(k)) \text { as } n \rightarrow \infty .
$$

To prove this, we apply the corresponding result of Jammalamadaka and Janson (1986) for independent samples. In fact, if we introduce the pooled random vectors in $R^{k d}$

$$
Y_{i}:=\left(X_{(i-1)(k+m)+1}, \ldots, X_{(i-1)(k+m)+k}\right), \quad i=1, \ldots, s,
$$

then the $m$-dependence of $\left\{X_{i}\right\}$ implies that $\left\{Y_{i}\right\}$ is an independent sequence in $R^{k d}$. Thus $U_{n}^{(k)}$ can be represented as a $U$-statistic with respect to the independent sample $Y_{1}, \ldots, Y_{s}$,

$$
W_{s}^{(k)}:=U_{n}^{(k)}=\sum_{1 \leq i<j \leq s} f_{s}^{(k)}\left(Y_{i}, Y_{j}\right), \quad f_{s}^{(k)}\left(Y_{i}, Y_{j}\right):=\sum_{l \in S_{i}^{(k)}} \sum_{t \in S_{j}^{(k)}} I\left(d\left(X_{l}, X_{t}\right) \leq \epsilon\right) .
$$

Furthermore, let $g_{s}^{(k)}\left(Y_{1}\right):=\mathrm{E}\left(f_{s}\left(Y_{1}, Y_{2}\right) \mid Y_{1}\right)=k \sum_{i=1}^{k} p_{X, \epsilon}\left(X_{i}\right)$ and define

$$
\eta_{s, k}^{2}:=\frac{1}{2} s^{2} \operatorname{Var}\left(f_{s}^{(k)}\left(Y_{1}, Y_{2}\right)\right)+s^{3} \operatorname{Var}\left(g_{s}^{(k)}\left(Y_{1}\right)\right)
$$

Since $n^{2} \epsilon^{d} \rightarrow \infty$ yields $s^{2} \epsilon^{d} \rightarrow \infty$, we obtain from Lemma 3 that

$$
\eta_{s, k}^{2} \geq \frac{1}{2} s^{2} \operatorname{Var}\left(f_{s}^{(k)}\left(Y_{1}, Y_{2}\right)\right) \sim \frac{1}{2} b_{1}(d) q_{2}(s k)^{2} \epsilon^{d} \rightarrow \infty \text { as } n \rightarrow \infty
$$

and therefore

$$
\sup _{y_{i}, y_{j}}\left|f_{s}^{(k)}\left(y_{i}, y_{j}\right)\right|=k^{2}=\mathrm{o}\left(\eta_{s, k}\right) \text { as } n \rightarrow \infty .
$$

Moreover, Jammalamadaka and Janson (1986) show that $\sup _{x} p_{X, \epsilon}(x)=\mathrm{o}\left(\epsilon^{d / 2}\right)$ as $\epsilon \rightarrow 0$, and hence the stationarity of $\left\{X_{i}\right\}$ and (39) give

$$
\sup _{y} \mathrm{E}\left|f_{s}^{(k)}\left(y, Y_{1}\right)\right| \leq k \sum_{i=1}^{k} \sup _{x} \mathrm{E} I\left(d\left(x, X_{i}\right) \leq \epsilon\right)=k^{2} \sup _{x} p_{X, \epsilon}(x)=\mathrm{o}\left(\epsilon^{d / 2}\right)=\mathrm{o}\left(\eta_{s, k} / s\right) \text { as } n \rightarrow \infty .
$$


Since $n \rightarrow \infty$ implies $s \rightarrow \infty$, we get from (40) and (41) that the conditions of Theorem 2.1 in Jammalamadaka and Janson (1986) are satisfied. Consequently

$$
\frac{\sigma_{n}}{\eta_{s, k}} Z_{n}^{(k)}=\frac{W_{s}^{(k)}-\mathrm{E} W_{s}^{(k)}}{\eta_{s, k}} \stackrel{\mathrm{D}}{\rightarrow} N(0,1) \text { as } n \rightarrow \infty .
$$

Further, using Proposition 1(ii) and definition (38), it is straightforward to show

$$
\eta_{s, k}^{2} / \sigma_{n}^{2} \rightarrow V(k) \text { as } n \rightarrow \infty
$$

so the desired limit (37) of $Z_{n}^{(k)}$ follows from (41) and the Slutsky theorem. This completes the proof.

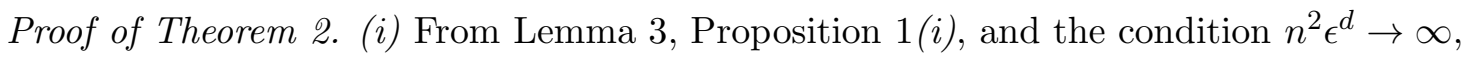

$$
\begin{aligned}
\mathrm{E} \tilde{Q}_{n} & =\tilde{q}_{2, \epsilon}+\mathrm{o}\left(\frac{1}{n \epsilon^{d / 2}}\right) \rightarrow q_{2}, \\
\operatorname{Var}\left(\tilde{Q}_{n}\right) & =\left(\begin{array}{c}
n \\
2
\end{array}\right)^{-2} b_{\epsilon}(d)^{-2} \sigma_{n}^{2}=\mathrm{O}\left(\frac{1}{n^{2} \epsilon^{d}}\right)+\mathrm{O}\left(\frac{1}{n}\right)+\mathrm{o}\left(\frac{1}{n^{3} \epsilon^{3 d / 2}}\right)+\mathrm{o}\left(\frac{1}{n^{2} \epsilon^{d}}\right) \\
& =\mathrm{O}\left(\frac{1}{n^{2} \epsilon^{d}}\right)+\mathrm{O}\left(\frac{1}{n}\right) \rightarrow 0 \text { as } n \rightarrow \infty .
\end{aligned}
$$

Hence, the assertion follows.

(ii) In order to avoid condition $\mathcal{A}$, we repeat the argument of Proposition $1(i)$ with the convergence rates in Lemma 3 (ii)-(iii) replaced by the weaker limits

$$
\sup _{\left\{i_{1}, i_{2}\right\} \neq\left\{j_{1}, j_{2}\right\}} P\left(d\left(X_{i_{1}}, X_{j_{1}}\right) \leq \epsilon, d\left(X_{i_{2}}, X_{j_{2}}\right) \leq \epsilon\right) \leq \sup _{i \neq j} P\left(d\left(X_{i}, X_{j}\right) \leq \epsilon\right) \rightarrow 0 \text { as } \epsilon \rightarrow 0,
$$

which follow from the stationarity and $m$-dependence of $\left\{X_{i}\right\}$ and since $P\left(X_{i}=X_{j}\right)=0$. First we obtain from (17) that

$$
\mu_{n}=\left(\begin{array}{l}
n \\
2
\end{array}\right) q_{2, \epsilon}+\mathrm{o}(n) \text { as } n \rightarrow \infty .
$$

Moreover, if we use (43) in place of Lemma 3 in the derivation of (19), (22), (23), and thus finally (26)), it follows that

$$
\begin{aligned}
& \operatorname{Var}\left(N_{n}^{*}\right)=\frac{n^{2}}{2} q_{2, \epsilon}+n^{3}\left(\sigma_{1,0, \epsilon}^{2}+2 \sum_{h=1}^{m} \sigma_{1, h, \epsilon}^{2}\right)+\mathrm{o}\left(n^{2}\right), \\
& \operatorname{Var}\left(N_{n}\right)=\operatorname{Var}\left(N_{n}^{*}\right)-\operatorname{Var}\left(N_{n}-N_{n}^{*}\right)+\mathrm{o}\left(n^{2}\right), \\
& \operatorname{Var}\left(N_{n}-N_{n}^{*}\right)=\mathrm{o}\left(n^{2}\right), \\
& \sigma_{n}^{2}=\frac{n^{2}}{2} q_{2, \epsilon}+n^{3}\left(\sigma_{1,0, \epsilon}^{2}+2 \sum_{h=1}^{m} \sigma_{1, h, \epsilon}^{2}\right)+\mathrm{o}\left(n^{2}\right), \text { as } n \rightarrow \infty .
\end{aligned}
$$

From (44), the last statement in (45), and the condition $n \epsilon^{d} \rightarrow a, 0<a \leq \infty$,

$$
\begin{aligned}
\mathrm{E} \tilde{Q}_{n} & =\tilde{q}_{2, \epsilon}+\mathrm{o}\left(\frac{1}{n \epsilon^{d}}\right) \rightarrow q_{2}, \\
\operatorname{Var}\left(\tilde{Q}_{n}\right) & =\left(\begin{array}{l}
n \\
2
\end{array}\right)^{-2} b_{\epsilon}(d)^{-2} \sigma_{n}^{2}=\mathrm{O}\left(\frac{1}{n^{2} \epsilon^{d}}\right)+\mathrm{O}\left(\frac{1}{n}\right)+\mathrm{o}\left(\frac{1}{n^{2} \epsilon^{2 d}}\right) \rightarrow 0 \text { as } n \rightarrow \infty,
\end{aligned}
$$


so the claim holds true. This completes the proof.

Proof of Theorem [3. (i) Let

$$
\sqrt{n}\left(\tilde{Q}_{n}-\tilde{q}_{2, \epsilon}\right)=\sqrt{n}\left(\begin{array}{l}
n \\
2
\end{array}\right)^{-1} b_{\epsilon}(d)^{-1}\left(N_{n}-\mathrm{E} N_{n}\right)+R_{n}
$$

where, by Proposition 1(i) and the assumption $n \epsilon^{d} \rightarrow a, 0<a \leq \infty$,

$$
R_{n}:=\sqrt{n}\left(\begin{array}{l}
n \\
2
\end{array}\right)^{-1} b_{\epsilon}(d)^{-1}\left(\mathrm{EN}_{n}-\left(\begin{array}{l}
n \\
2
\end{array}\right) q_{2, \epsilon}\right)=\mathrm{o}\left(\frac{1}{\sqrt{n \epsilon^{d}}}\right) \rightarrow 0 \text { as } n \rightarrow \infty .
$$

Furthermore, from Proposition 1 (ii),

$$
\left(\sqrt{n}\left(\begin{array}{l}
n \\
2
\end{array}\right)^{-1} b_{\epsilon}(d)^{-1}\right)^{2} \sigma_{n}^{2} \rightarrow \nu / a+4 \zeta_{1, m} \text { as } n \rightarrow \infty .
$$

Finally, combining (46), (47), (48), Theorem 1(iii), and the Slutsky theorem gives the assertion for $\tilde{Q}_{n}$. The statement about $\tilde{H}_{n}$ follows from Proposition 2 in Leonenko and Seleznjev (2010).

(ii) The details are omitted, since the argument is similar to that of (i), using the decomposition corresponding to (46) with the $n \epsilon^{d / 2}$-scaling. This completes the proof.

Proof of Theorem 4. (i) As in Leonenko and Seleznjev (2010), the density smoothness condition yields

$$
\left|\tilde{q}_{2, \epsilon}-q_{2}\right| \leq \frac{1}{2} K^{2} \epsilon^{2 \alpha} .
$$

This bound and Proposition 1(i) imply the assertion

$$
\left|\mathrm{E} \tilde{Q}_{n}-q_{2}\right| \leq\left|\tilde{q}_{2, \epsilon}-q_{2}\right|+\left|\mathrm{E} \tilde{Q}_{n}-\tilde{q}_{2, \epsilon}\right| \leq \frac{1}{2} K^{2} \epsilon^{2 \alpha}+\mathrm{o}\left(1 /\left(n \epsilon^{d / 2}\right)\right) \text { as } n \rightarrow \infty .
$$

(ii) Note that, by the assumptions $\epsilon \sim c n^{-2 /(4 \alpha+d)}$ and $0<\alpha \leq d / 4$,

$$
n^{2} \epsilon^{d} \sim c^{d} n^{8 \alpha /(4 \alpha+d)} \leq c^{d} n \text { as } n \rightarrow \infty
$$

and hence, from (42),

$$
\operatorname{Var}\left(\tilde{Q}_{n}\right)=\mathrm{O}\left(n^{-8 \alpha /(4 \alpha+d)}\right) \text { as } n \rightarrow \infty .
$$

Further, since $\epsilon^{2 \alpha} \sim c^{2 \alpha} n^{-4 \alpha /(4 \alpha+d)}$, we get from (i) and (50) that the bias fulfills

$$
\left|\mathrm{E} \tilde{Q}_{n}-q_{2}\right|=\mathrm{O}\left(n^{-4 \alpha /(4 \alpha+d)}\right) \text { as } n \rightarrow \infty .
$$

Consequently, for some $C>0$ and any $A>0$,

$$
P\left(\left|\tilde{Q}_{n}-q_{2}\right|>A n^{-4 \alpha /(4 \alpha+d)}\right) \leq n^{8 \alpha /(4 \alpha+d)} \frac{\operatorname{Var}\left(\tilde{Q}_{n}\right)+\left(\mathrm{E} \tilde{Q}_{n}-q_{2}\right)^{2}}{A^{2}} \leq \frac{C}{A^{2}}
$$

and the desired convergence for $\tilde{Q}_{n}$ follows. Moreover, combining this with Proposition 2 in Leonenko and Seleznjev (2010) proves the statement for $\tilde{H}_{n}$. 
(iii) The argument is similar to that of (ii) and therefore is left out. This completes the proof.

Proof of Proposition [2. First we study the expectation of $U_{h, n}$. By Lemma 1, the number of 3tuples $\left(s_{1}, s_{2}, s_{3}\right)$ that satisfy $s_{i+1}-s_{i}>h+m, i=1,2$, is $\left(\begin{array}{c}n-2(m+h) \\ 3\end{array}\right)$. Furthermore, observe that 3 ! of the elements $(i, j, k) \in \mathcal{E}_{h, n}$ are permutations of $\left(s_{1}, s_{2}, s_{3}\right)$. For the corresponding variables, $X_{j}$ and $X_{k}$ are mutually independent and also independent of $\left\{X_{i}, X_{i+h}\right\}$, so for these we obtain

$$
\mathrm{E} I\left(d\left(X_{i}, X_{j}\right) \leq \epsilon_{0}, d\left(X_{i+h}, X_{k}\right) \leq \epsilon_{0}\right)=\mathrm{E}_{p_{X, \epsilon_{0}}}\left(X_{i}\right) p_{X, \epsilon_{0}}\left(X_{i+h}\right)=\mathrm{E} p_{X, \epsilon_{0}}\left(X_{1}\right) p_{X, \epsilon_{0}}\left(X_{1+h}\right) .
$$

Thus, from Lemma 3 and the assumption $n \epsilon_{0}^{d} \rightarrow \infty$,

$$
\begin{aligned}
\mathrm{E} U_{h, n} & =3 !\left(\begin{array}{c}
n-2(m+2 h) \\
3
\end{array}\right) M_{h, n}^{-1} b_{\epsilon_{0}}(d)^{-2} \mathrm{E} p_{X, \epsilon_{0}}\left(X_{1}\right) p_{X, \epsilon_{0}}\left(X_{1+h}\right)+\mathrm{o}\left(1 /\left(n \epsilon_{0}^{d}\right)\right) \\
& \rightarrow \mathrm{E} p\left(X_{1}\right) p\left(X_{1+h}\right)=q_{3, h} \text { as } n \rightarrow \infty
\end{aligned}
$$

Next we consider the variance of $U_{h, n}$. Using the notation $I_{\epsilon_{0}}(i, j):=I\left(d\left(X_{i}, X_{j}\right) \leq \epsilon_{0}\right)$, we have

$$
\operatorname{Var}\left(U_{h, n}\right)=M_{h, n}^{-2} b_{\epsilon_{0}}(d)^{-4} \sum_{\substack{\left(s_{1}, s_{2}, s_{3}\right) \in \mathcal{E}_{h, n} \\\left(t_{1}, t_{2}, t_{3}\right) \in \mathcal{E}_{h, n}}} \operatorname{Cov}\left(I_{\epsilon_{0}}\left(s_{1}, s_{2}\right) I_{\epsilon_{0}}\left(s_{1}+h, s_{3}\right), I_{\epsilon_{0}}\left(t_{1}, t_{2}\right) I_{\epsilon_{0}}\left(t_{1}+h, t_{3}\right)\right) .
$$

We count the number of terms in this sum that are zero. Lemma 1 implies that the number of 6-tuples $\left\{u_{1}, \ldots, u_{6}\right\} \subseteq\{1, \ldots, n\}$ with $u_{i+1}-u_{i}>m+h, i=1, \ldots, 5$, is $\left(\begin{array}{c}n-5(m+h) \\ 6\end{array}\right)$. Each such 6 -tuple can be divided and permuted into $\left(\begin{array}{l}6 \\ 3\end{array}\right) \times 3 ! \times 3 !=6$ ! pairs $\left(s_{1}, s_{2}, s_{3}\right),\left(t_{1}, t_{2}, t_{3}\right) \in \mathcal{E}_{h, n}$. The $m$-dependence of $\left\{X_{i}\right\}$ yields that the corresponding random variables are independent, and hence at least

$$
6 !\left(\begin{array}{c}
n-5(m+h) \\
6
\end{array}\right)=M_{h, n}^{2}+\mathrm{O}\left(n^{5}\right) \text { as } n \rightarrow \infty
$$

summands in (52) are zero. For each of the $\mathrm{O}\left(n^{5}\right)$ non-zero terms,

$$
\begin{aligned}
\mid \operatorname{Cov}\left(I_{\epsilon_{0}}\left(s_{1}, s_{2}\right) I_{\epsilon_{0}}\left(s_{1}+h, s_{3}\right)\right. & \left.I_{\epsilon_{0}}\left(t_{1}, t_{2}\right) I_{\epsilon_{0}}\left(t_{1}+h, t_{3}\right)\right) \mid \\
& \leq\left(\mathrm{E} I_{\epsilon_{0}}\left(s_{1}, s_{2}\right) I_{\epsilon_{0}}\left(s_{1}+h, s_{3}\right)\right)^{1 / 2}\left(\mathrm{E} I_{\epsilon_{0}}\left(t_{1}, t_{2}\right) I_{\epsilon_{0}}\left(t_{1}+h, t_{3}\right)\right)^{1 / 2},
\end{aligned}
$$

so Lemma 3(iii) gives that the sum of the non-zero terms in (52) is o $\left(n^{5} \epsilon_{0}^{d}\right)$ as $n \rightarrow \infty$. Combining this with the condition $n \epsilon_{0}^{3 d} \rightarrow c, 0<c \leq \infty$, we get

$$
\operatorname{Var}\left(U_{h, n}\right)=\mathrm{o}\left(\frac{1}{n \epsilon_{0}^{3 d}}\right) \rightarrow 0 \text { as } n \rightarrow \infty
$$

Finally, from (51) and (53) it follows that $\mathrm{E}\left(U_{h, n}-q_{3, h}\right)^{2} \rightarrow 0$, which completes the proof.

Proof of Theorem 5. The argument is similar to that of Theorem 6 in Leonenko and Seleznjev (2010), so we show the main steps only. From the decomposition

$$
\sqrt{n}\left(\tilde{Q}_{n}-q_{2}\right)=\sqrt{n}\left(\tilde{Q}_{n}-\tilde{q}_{2, \epsilon}\right)+\sqrt{n}\left(\tilde{q}_{2, \epsilon}-q_{2}\right)
$$


we see that the assertion for $\tilde{Q}_{n}$ is implied by the Slutsky theorem if $\sqrt{n}\left(\tilde{Q}_{n}-\tilde{q}_{2, \epsilon}\right) \stackrel{\mathrm{D}}{\rightarrow} N\left(0, \nu / a+\zeta_{1, m}\right)$ and $\left|\sqrt{n}\left(\tilde{q}_{2, \epsilon}-q_{2}\right)\right| \rightarrow 0$. The asymptotic normality follows straight away from Theorem $3(i)$. Furthermore, the conditions for $\alpha$ and $\epsilon$ together with bound (49) lead to the desired convergence of $\left|\sqrt{n}\left(\tilde{q}_{2, \epsilon}-q_{2}\right)\right|$. Finally, Proposition 2 in Leonenko and Seleznjev (2010) proves the claim for $\tilde{H}_{n}$. This completes the proof.

Proof of Theorem [6. (i) We use the decomposition corresponding to (54):

$$
n^{\beta / 2} c^{d / 2}\left(\tilde{Q}_{n}-q_{2}\right)=n^{\beta / 2} c^{d / 2}\left(\tilde{Q}_{n}-\tilde{q}_{2, \epsilon}\right)+n^{\beta / 2} c^{d / 2}\left(\tilde{q}_{2, \epsilon}-q_{2}\right) .
$$

Note that the condition $\epsilon \sim c n^{-(2-\beta) / d}$ gives $n \epsilon^{d} \rightarrow 0$ and $n^{\beta / 2} c^{d / 2} /\left(n \epsilon^{d / 2}\right) \rightarrow 1$, so the asymptotic normality

$$
n^{\beta / 2} c^{d / 2}\left(\tilde{Q}_{n}-\tilde{q}_{2, \epsilon}\right) \stackrel{\mathrm{D}}{\rightarrow} N(0, \nu) \text { as } n \rightarrow \infty
$$

follows from Theorem 3 (ii) and the Slutsky theorem. Further, the assumptions $\alpha>(d / 4) C_{\beta}$, $\epsilon \sim c n^{-(2-\beta) / d}$, and bound (49) imply

$$
\left|n^{\beta / 2} c^{d / 2}\left(\tilde{q}_{2, \epsilon}-q_{2}\right)\right| \leq c^{d / 2} \frac{1}{2} K^{2} n^{\beta / 2} \epsilon^{2 \alpha} \sim c^{d / 2+2 \alpha} \frac{1}{2} K^{2} n^{\beta / 2-2 \alpha(2-\beta) / d} \rightarrow 0 \text { as } n \rightarrow \infty,
$$

since $\beta / 2-2 \alpha(2-\beta) / d<\beta / 2-2(d / 4) C_{\beta}(2-\beta) / d=0$. Thus, from (55), (56), (57), and the Slutsky theorem, we obtain the statement for $\tilde{Q}_{n}$. The assertion for $\tilde{H}_{n}$ follows by an argument similar to that of Proposition 2 in Leonenko and Seleznjev (2010).

(ii) The argument follows the same steps as that of (i) and consequently is omitted. This completes the proof. 


\section{References}

Anderson, T.W. (1971), The Statistical Analysis of Time Series, New York: John Wiley and Sons.

Barbour, A.D., Holst, L., Janson, S. (1992), Poisson Approximation, Oxford: Oxford University Press.

Baryshnikov, Y., Penrose, M.D., Yukich, J.E. (2009), 'Gaussian limits for generalized spacings', Ann. Appl. Probab., 19, 158-185.

Bickel, P.J. and Ritov, Y. (1988), 'Estimating integrated squared density derivatives: sharp best order of convergence estimates', Sankhyā: The Indian Journal of Statistics, Series A, 381-393.

Billlingsley, P. (1995), Probability and Measure, New York: Wiley.

Escolano, F., Suau, P., Bonev, B. (2009), Information Theory in Computer Vision and Pattern Recognition, New York: Springer.

Giné, E., Nickl, R. (2008), 'A simple adaptive estimator for the integrated square of a density', Bernoulli, $14,47-61$.

Goria, M.N., Leonenko, N.N., Mergel, V.V., Inverardi, P.L.N. (2005), 'A new class of random vector entropy estimators and its applications in testing statistical hypotheses', J. Nonparam. Stat., 17, $277-297$.

Gregorio, A., Iacus, S.M. (2009), 'On Rényi information for ergodic diffusion processes', Inform. Sci., 179, $279-291$.

Harrelson, D., Houdré, C. (2003), 'A characterization of $m$-dependent stationary infinitely divisible sequences with applications to weak convergence', Ann. Probab., 31, 849-881.

Hart, J.D., Vieu, P. (1990), 'Data-driven bandwidth choice for density estimation based on dependent data', Ann. Stat., 18, 873-890.

Jammalamadaka, S.R., Janson, S. (1986), 'Limit theorems for a triangular scheme of $U$-statistics with applications to inter-point distances', Ann. Probab., 14, 1347-1358.

Johnson, O., Vignat C. (2007), 'Some results concerning maximum Rényi entropy distributions', Ann. Inst. H. Poincaré Probab. Statist., 43, 339-351.

Joe, H. (1997), Multivariate Models and Dependence Concepts, London: Chapman and Hall.

Kapur, J.N. (1989), Maximum-entropy Models in Science and Engineering, New York: Wiley.

Kapur, J.N., Kesavan, H.K. (1992), Entropy Optimization Principles with Applications, New York: Academic Press.

Kim, T.Y., Luo, Z.M., Kim, C. (2011), 'The central limit theorem for degenerate variable $U$-statistics under dependence', J. Nonparam. Stat., 23, 683-699.

Koroljuk, V.S., Borovskich, Y.V. (1994), Theory of U-statistics, Dordrecht: Kluwer.

Kotz, S., Balakrishnan, N., Johnson, N.L. (2000), Continuous multivariate distributions: Models and applications, New York: Wiley.

Källberg, D., Seleznjev, O. (2012), 'Estimation of entropy-type integral functionals', preprint arXiv:1209.2544.

Källberg, D., Leonenko, N., Seleznjev, O. (2012), 'Statistical inference for Rényi entropy functionals', Lecture Notes in Comput. Sci., 7260, 36-51.

Last, Y., Klein, M., Kandel, A. (2001), 'Knowledge discovery in time series databases', IEEE Trans. Systems. Man. and Cybernetics - Part B, 31, 160-169. 
Laurent, B. (1996), 'Efficient estimation of integral functionals of a density', Ann. Statist., 24, 659-681.

Lee, A.J. (1990), U-Statistics: Theory and Practice, New York: Marcel Dekker.

Leonenko, N., Pronzato, L., Savani, V. (2008), 'A class of Rényi information estimators for multidimensional densities', Ann. Statist., 36, 2153-2182. Corrections, (2010), Ann. Statist., 38, 3837-3838.

Leonenko, N., Seleznjev, O. (2010), 'Statistical inference for the $\epsilon$-entropy and the quadratic Rényi entropy', J. Multivariate Anal., 101, 1981-1994.

Neemuchwala, H., Hero, A., Carson, P. (2005), 'Image matching using alpha-entropy measures and entropic graphs', Signal Processing, 85, 277-296.

Pardo, L. (2006), Statistical Inference Based on Divergence Measures, Boca Raton: Chapman \& Hall.

Penrose, M., Yukich, J.E. (2011), 'Limit theory for point processes in manifolds', Annals of Applied Probability, to appear, see also preprint arXiv:1104.0914 1 .

Principe, J.C. (2010), Information Theoretic Learning, New York: Springer.

Rényi, A. (1970), Probability Theory, Amsterdam: North-Holland.

Seleznjev, O., Thalheim, B. (2003), 'Average case analysis in database problems', Methodol. Comput. Appl. Prob., 5, 395-418.

Seleznjev, O., Thalheim, B. (2010), 'Random databases with approximate record matching', Methodol. Comput. Appl. Prob., 12, 63-89.

Serfling, R.J. (2002), Approximation Theorems of Mathematical Statistics, New York: Wiley.

Shannon, C.E. (1948), 'A mathematical theory of communication', Bell Syst. Tech. Jour., 27, 379-423, 623-656.

Thalheim, B. (2000), Entity-Relationship Modeling. Foundations of Database Technology, Berlin: SpringerVerlag.

Tsallis, C. (1988), 'Possible generalization of Boltzmann-Gibbs statistics', J. Stat. Phys., 52, 479-487.

Ullah, A. (1996), 'Entropy, divergence and distance measures with econometric applications'. J. Statist. Plann. Inference, 49, 137-162.

Vatutin,V. A., Mikhailov, V.G. (1995), 'Statistical estimation of the entropy of discrete random variables with a large number of outcomes', Russian Math. Surveys, 50, 963-976.

Wang, Q. (1999), 'On Berry-Esseen rates for $m$-dependent $U$-statistics', Stat. Prob. Letters, 41, 123-130.

\section{Appendix. Estimation for discrete distributions}

Consider a stationary $m$-dependent sequence $\left\{X_{1}, \ldots, X_{n}\right\}$ with discrete $d$-dimensional (marginal) distribution $\mathcal{P}=\left\{p(k), k \in N^{d}\right\}$. We present some results on the estimation of quadratic Rényi entropy for discrete distributions

$$
h_{2}(\mathcal{P}):=-\log \left(\sum_{k} p(k)^{2}\right)=-\log \left(q_{2}\right)
$$

and the corresponding quadratic functional

$$
q_{2}:=\sum_{k} p(k)^{2}=P(X=Y),
$$


where $X$ and $Y$ are independent vectors with distribution $\mathcal{P}$. Similarly to the continuous case, let

$$
\zeta_{1, m}:=\operatorname{Var}\left(p\left(X_{1}\right)\right)+2 \sum_{h=1}^{m} \operatorname{Cov}\left(p\left(X_{1}\right), p\left(X_{h+t}\right)\right) .
$$

Define the normalized statistic

$$
Q_{n}:=\left(\begin{array}{l}
n \\
2
\end{array}\right)^{-1} \sum_{1 \leq i<j \leq n} I\left(X_{i}=X_{j}\right)=\left(\begin{array}{l}
n \\
2
\end{array}\right)^{-1} N_{n, 0}
$$

to be an estimator for $q_{2}$. Let $H_{n}:=-\log \left(\max \left(Q_{n}, 1 / n\right)\right)$ be the corresponding estimator for $h_{2}$. For $h=0, \ldots, m$, we also introduce the following estimator for $q_{3, h}:=\mathrm{E} p\left(X_{1}\right) p\left(X_{1+h}\right)$,

$$
U_{h, n}:=M_{h, n}^{-1} \sum_{(i, j, k) \in \mathcal{E}_{h, n}} I\left(X_{i}=X_{j}=X_{i+h}=X_{k}\right)
$$

where $\mathcal{E}_{n, h}$ and $M_{n, h}$ are defined as in Section 2, By an argument similar to that of Proposition 2, we get $U_{h, n} \stackrel{\mathrm{P}}{\rightarrow} q_{3, h}$. Hence, for $r \geq m$, a consistent estimator for $\zeta_{1, m}$ is given by

$$
s_{r, n}^{2}:=U_{0, n}-Q_{n}^{2}+2 \sum_{i=1}^{r}\left(U_{h, n}-Q_{n}^{2}\right) .
$$

Some asymptotic properties for the estimators of $q_{2}$ and $h_{2}$ follow by combining the results of Ch. 2 in Lee (1990), Wang (1999), and the Slutsky theorem.

\section{Theorem 7}

(i) For the expectation and variance, we obtain

$$
\begin{aligned}
\mathrm{E}\left(Q_{n}\right) & =q_{2}+\mathrm{O}\left(n^{-1}\right), \\
\operatorname{Var}\left(Q_{n}\right) & =4 \zeta_{1, m} n^{-1}+\mathrm{O}\left(n^{-2}\right) \text { as } n \rightarrow \infty,
\end{aligned}
$$

and thus $Q_{n}$ and $H_{n}$ are consistent estimators for $q_{2}$ and $h_{2}$, respectively.

(ii) If $\zeta_{1, m}>0$ and $r \geq m$, then

$$
\begin{aligned}
& \sqrt{n}\left(Q_{n}-q_{2}\right) \stackrel{\mathrm{D}}{\rightarrow} N\left(0,4 \zeta_{1, m}\right) \quad \text { and } \quad \frac{\sqrt{n}}{2 s_{r, n}}\left(Q_{n}-q_{2}\right) \stackrel{\mathrm{D}}{\rightarrow} N(0,1) ; \\
& \frac{\sqrt{n}}{2 s_{r, n}} Q_{n}\left(H_{n}-h_{2}\right) \stackrel{\mathrm{D}}{\rightarrow} N(0,1) \text { as } n \rightarrow \infty .
\end{aligned}
$$

As in the continuous case, we have $\zeta_{1, m} \geq 0$ with equality, e.g., if $\mathcal{P}$ is uniform. 